\title{
ORGANIC CHEMICAL APPLICATIONS OF MAGNETIC CIRCULAR DICHROISM*
}

\author{
Carl Djerassi, Edward Bunnenberg and Danny L. Elder
}

Department of Chemistry, Stanford University, Stanford, California

\begin{abstract}
Recent applications of magnetic circular dichroism spectroscopy (the Faraday effect) in organic chemistry are reviewed. Magnetic optical activity is a universal property of all matter and consequently can be applied to optically active as well as optically inactive molecules. It is emphasized, however, that natural and magnetic optical activity have separate physical origins and the information provided will necessarily be different. A brief theoretical background is given in order to explain the three types (A, B and C) of MCD curves which may be encountered in practice and the deductions that may be reached from their occurrence. Spectroscopic and analytical applications of MCD are illustrated for a wide variety of molecular systems such as metalloporphyrins, chlorins, corrins, aromatic hydrocarbons, carbonyl compounds, purine and pyrimidine bases and their nucleoside derivatives.
\end{abstract}

\section{INTRODUCTION}

The natural product chemist has been particularly receptive to the use of new physical techniques. Indeed in many instances, major advances in methodology arose from semi-empirical studies performed by organic chemists with model compounds (e.g. carotenoids and steroids in ultraviolet spectroscopy, steroid ketones in infra-red spectroscopy, fatty acids and indole alkaloids in mass spectrometry, etc.) derived from the natural product field. In each instance, the method provided information which was either unavailable or else only difficultly extractable by other means and the judicious use of a combination of physical methods is a conditio sine qua non for the modern organic or biological chemist. The actual number of different physical techniques employed routinely by the organic chemist is small, principally because permanent addition to his experimental armamentarium requires either that new information be provided or that known data be presented in a more convenient form.

Optical rotatory dispersion and circular dichroism are two closely related physical methods, whose importance in organic chemical methodology was first demonstrated as a result of work performed in the steroid field. Ten years ago one ${ }^{1}$ of the plenary lectures at the first IUPAC International Symposium on the Chemistry of Natural Products dealt with applications of optical rotatory dispersion studies to problems in natural products chemistry. The date of that lecture was only a few months after the appearance of the

* Paper XV in the series 'Magnetic circular dichroism studies'. 
first modern monograph ${ }^{2}$ on organic chemical applications of optical rotatory dispersion and it was still an easy matter to review the subject exhaustively. Four years later, in a Centenary Lecture ${ }^{3}$ attention was called to the explosive growth of the field and the appearance in those intervening years of over 700 articles dealing with organic chemical applications of optical rotatory dispersion and circular dichroism. Since then, several books ${ }^{4-6}$ have appeared which have barely kept up with the progress and there is no question that both techniques have found a secure place in organic chemical methodology.

The most important uses to which these two methods have been put are in the field of stereochemistry, with special emphasis on the determination of absolute configuration and on conformational analysis. In addition, there have been numerous analytical applications, notably in kinetic studies, which have taken advantage of the greatly increased rotation or ellipticity values in the spectral region of maximum absorption (see Figure 1).

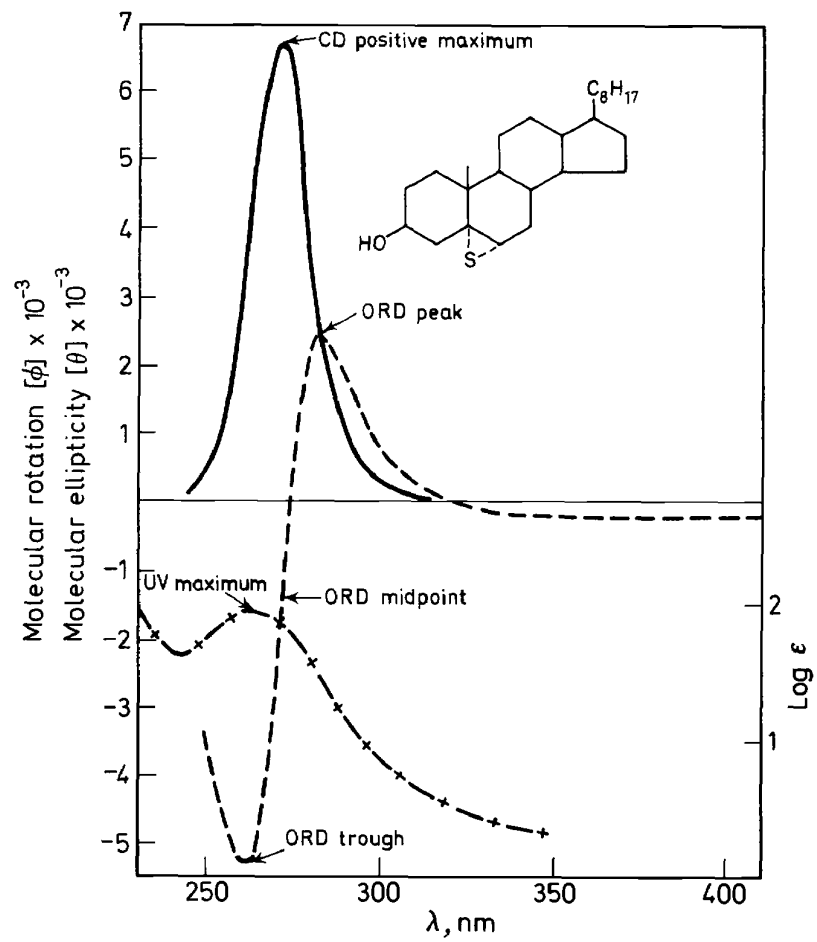

Figure 1. Illustration of the relation between optical rotatory dispersion, circular dichroism and absorption spectra ( $3 \beta$-hydroxycholestan- $5 \alpha, 6 \alpha$-episulphide).

The data in Figure $1^{3}$ illustrate the well-known relation between absorption, circular dichroism and optical rotatory dispersion and also point to two limiting requirements. The first is an absolute one and refers to the necessity of natural optical activity in the substance under investigation. Fortunately, this requirement does not impose major limitations among 
natural products, because the overwhelming majority of them are optically active. It does, however, eliminate the majority of synthetic organic chemicals. The second requirement, which is indispensable for circular dichroism and very important in optical rotatory dispersion is that the substance exhibit one or more absorption bands in an accessible spectral region (in general above $190 \mathrm{~nm}$ ) and that the intensity of the absorption band not be so high as to preclude measurement of the circular dichroism or rotatory dispersion. The existence of an absorption maximum is not an absolute requirement in optical rotatory dispersion, because optical rotation will also be observed outside the region of absorption; however, the resulting curve will only be of the 'plain' type ${ }^{7}$ (e.g. $300-400 \mathrm{~nm}$ region in Figure 1) and hence will not offer the wealth of information that is often extractable from curves displaying a Cotton effect (e.g. 250-300 $\mathrm{nm}$ region in Figure 1).

The purpose of this plenary lecture, which follows almost exactly by ten years the first one ${ }^{1}$ dealing with optical rotatory dispersion, is to examine the possibility of extending rotatory dispersion and circular dichroism techniques to optically inactive substances. A successful approach is provided by Faraday's discovery in the last century ${ }^{8}$ that a substance placed in a magnetic field rotates the plane of polarized light-a phenomenon since known as the 'Faraday effect'. Of crucial importance to our subsequent discussion and to the selection of the optimum experimental conditions is the fact that all materials, irrespective of their chemical or geometrical structure, exhibit rotation in a magnetic field.

Verdet ${ }^{9}$ was the first to express the Faraday effect in quantitative terms through the expression $\alpha=\delta l H \cos \theta$, in which $\alpha$ is the magnetic rotation, $l$ the path length of light, $H$ the magnetic field strength and $\theta$ the angle between the magnetic lines of force and the optical beam. The proportionality constant $\delta$ is now known as Verdet's constant and is characteristic (at a given wavelength) of the particular substance. Since the magnetic field and the light beam are usually parallel, $\cos \theta$ equals unity and hence can usually be omitted. Just as in the early development of optical rotatory dispersion ${ }^{2}$, most of the initial magneto-optical measurements were performed in the visible region outside the range of absorption bands. In the case of organic molecules, principal attention was paid to measurements of Verdet constants, and mainly through the efforts of Perkin ${ }^{10}$ extensive correlations between magnetic rotatory power (usually determined at the sodium D line) and constitution were attempted in order to obtain additivity relations similar to those observed in the use of molecular refractivity. A review ${ }^{11}$ of the experimental and theoretical state of magnetic rotatory power until the early 1930 s discloses that hardly any progress has been made in organic chemical applications since Perkin's Verdet constant measurements during the last century. In other words, this phase of magnetic optical investigations can be equated in terms of natural optical activity to the early emphasis on plain dispersion curves, i.e. studies outside the region of absorption. Such measurements are of importance now only in Faraday effect investigations of hydrocarbons ${ }^{12,13}$.

The first significant experimental observation on the existence of anomalous magnetic rotatory dispersion was made around the turn of the century by Wood ${ }^{14}$ who carried out measurements through one of the absorption 


\section{CARL DJERASSI, EDWARD BUNNENBERG AND DANNY ELDER}

bands of praseodymium chloride. The first such observation with an organic compound was made only in 1932, when Cotton and Scherer ${ }^{15}$ described the magnetic rotatory dispersion curve of thiobenzophenone in the region (i.e. on either side) of its $600 \mathrm{~nm}$ absorption band. Ten years later, similar measurements were performed ${ }^{16}$ through the $n-\pi^{*}$ absorption band of 2-pentanone but thereafter no significant research activity occurred until the early 1960 s. It is precisely with such research that we should like to occupy ourselves in this article.

It is not surprising that the renewed interest in optical rotatory dispersion and circular dichroism of organic compounds which commenced about 15 years ago should also have had repercussions in the area of Faraday effect measurements. As has been the case in all other developments involving the use of physical methods by organic chemists, one of the most important incentives is invariably the existence of convenient instrumentation. Thus, as pointed out earlier ${ }^{2}$, the introduction of the spectropolarimeter was the single most important factor that allowed the organic chemist to contribute so heavily to modern advances in optical rotatory dispersion and circular dichroism. Once many such instruments became available, then all that was really necessary was to incorporate a suitable magnet in order to extend these optical measurements to their magnetic counterparts. Instrumentally, the developments in the optical rotatory dispersion field ${ }^{2}$ preceded those $^{3-6}$ in the circular dichroism area and it is not surprising, therefore, that the same chronology was followed with Faraday effect measurements of organic compounds. The first significant study was recorded by Shashoua ${ }^{17}$ who inserted an electromagnet, capable of a magnetic field strength of up to 18 kgauss, into a spectropolarimeter and measured the magnetic rotatory dispersion of eleven optically inactive organic compounds (e.g. acetone, furan, acridine, ethylene trithiocarbonate, etc.) through their region of absorption. Several laboratories, including our own, had initiated Faraday effect measurements of organic compounds around that time and it is instructive to note why eventually the major emphasis was placed on magnetic circular dichroism (henceforth abbreviated as MCD) rather than on magnetic optical rotation (abbreviated as MOR).

Figure 1 illustrates the well-known fact ${ }^{2-5}$ that the natural circular dichroism of an optically active molecule is only observed in the region of its absorption band, whereas optical rotation is encountered in that region as well as far away from it. Circular dichroism curves, therefore, are simpler in shape and are especially advantageous in substances with closely spaced absorption bands. Rotatory dispersion, on the other hand, because of its operation outside the region of maximal absorption, has the advantage of being measurable in compounds whose absorption is either outside the measurable spectral range or else is too intense for feasible signal detection. Furthermore, the greater complexity of ORD curves resulting from the frequent superposition of background rotational effects and Cotton effects may also offer important advantages ${ }^{3}$ for 'finger printing' purposes. In principle, precisely the same statements can also be made for MCD and MOR.

However, an additional factor needs to be considered in Faraday effect measurements, which does not exist in $\mathrm{CD}$ and ORD. Exposure to a magnetic 
field induces optical activity in all molecules, including the solvent, and since the latter is present in much greater concentration than the solute, the signal associated with the solute is frequently difficult to measure. When measurements have to be carried through the region of absorption of the solute, the solution may have to be particularly dilute and if the 'magnetic Cotton effect' (a misnomer as shown below) is weak, it may be very difficult to determine it with any type of precision. None of these difficulties exist in MCD, provided a solvent is employed that is optically transparent in the absorption region of the solute.

Instrumentally ${ }^{18}$, the complications in MOR measurements associated with the optical activity of the solvent can be overcome by incorporating into the spectropolarimeter in tandem two identical magnets (either solenoids or permanent magnets), which are arranged so that the fields oppose each other. The first magnet contains a sample tube with pure solvent, whereas an identical sample tube containing the solution is placed into the second magnet. Since the two magnetic fields are of identical strength but opposite direction, the contribution of the solvent cancels out and the observed magnetically induced rotation should be attributable only to the solute. This is the technique employed by us in our initial MOR studies ${ }^{19}$ and by others $^{18,20}$.

The final and decisive factor, which led us $^{21}$ to concentrate exclusively on MCD, was the desire to use as high a magnetic field as possible, since the intensity of the signal is proportional to the strength of the magnetic field. Such a requirement is particularly important for compounds (e.g. ketones), which display weak but very interesting signals, or for certain analytical purposes (e.g. analysis of lunar samples). Experimentally, the answer is provided $^{21}$ by superconducting magnets which provide fields in excess of 50 kgauss and yet are sufficiently small in size so as to be easily inserted into commercially available dichrometers. Their initial cost as well as consumption of liquid helium realistically precludes their use in a tandem arrangement for MOR measurements.

Superficially, CD and MCD or ORD and MOR would appear to be closely related, since in each instance one is dealing either with differences in absorption (circular dichroism) or refractive index (rotation) for right and left circularly polarized light. Various relations such as the KronigKramers theorem ${ }^{22}$ or the Drude equation ${ }^{23}$ apply equally to natural optical and magnetic optical parameters; similarly, the data in each case are expressed in identical ways (e.g. [ $\theta]$ or $\Delta \varepsilon$ in CD and MCD) since one is dealing either with differences in absorption $\left(\varepsilon_{L}-\varepsilon_{R}\right)$ or refractive index $\left(n_{\mathrm{L}}-n_{\mathrm{R}}\right)$. In addition, the sign convention is the same for both natural and magnetic optical activity provided that the light beam is moving in the positive direction of the magnetic field ${ }^{24,25}$. Nevertheless, there exists a fundamental difference between natural and magnetically induced optical activity. In the former, the disturbing factor is internal to the molecule and its own dissymmetry is responsible for the fact that upon excitation the electrons follow a helical path with the chirality of the helix being responsible for the differential absorption of right and left circularly polarized light. In magnetically induced optical activity, the disturbing factor (i.e. magnetic field) is now external to the molecule and the latter's geometry should not 
play an important role, whereas molecular geometry is crucial to ORD and $C D$. In the case of the Faraday effect, the right and left circularly polarized photons no longer interact equivalently with the absorbing medium under the influence of the magnetic field.

In a highly significant article, Buckingham and Stephens ${ }^{24}$ reviewed the theory of magnetic optical activity and more recently, another important review $^{25}$ appeared covering both theory and recent developments in inorganic chemistry. These articles eliminate the necessity for covering theoretical aspects in the present contribution, whereas the very brief mention of MCD applications in organic chemistry by previous reviewers ${ }^{24,25}$ justifies our concentration upon this topic. In discussing the experimental results, we shall concentrate mainly on contributions from our own laboratory, but before doing so a short coverage of the effect of a magnetic field upon electronic states is indicated in order to explain the three types (A, B and C) of MCD curves that may be encountered in practice and the deductions that may be reached from their occurrence.

\section{GENERAL BACKGROUND TO MAGNETIC CIRCULAR DICHROISM}

Already at an early stage, theoreticians noted the analogy between the Faraday and the Zeeman effect. The normal longitudinal Zeeman effect is characterized as the splitting of a spectral line into a left and a right circularly polarized component. The Faraday effect was then treated theoretically by considering the longitudinal Zeeman effect in absorption. Serber ${ }^{26}$ showed that three terms were involved in calculating the Faraday rotation of a molecule: the $\boldsymbol{A}$ term is related to the Zeeman splitting of the spectral lines into a left and a right circularly polarized component; the $B$ term is due to perturbations in the energy levels caused by the magnetic field; and the $C$ term is associated with a change in electronic population of the split ground state. Serber's nomenclature has been retained by Buckingham and Stephens ${ }^{24}$ in their quantum mechanical calculations as well as by Schatz and $\mathrm{McCaffer}^{25}$. The simple diagrammatic representations encompassed in Figures 2-4 are relatively minor modifications of those employed by Schatz and McCaffery ${ }^{25}$.

It is convenient to consider an isolated electronic transition in which an electron is promoted from a ground state $a$ to an excited state $j$ [Figure 2(a)]. If the incident light beam is randomly polarized, one observes an absorption band at the resonance frequency $v_{0}$ as shown in Figure 2(b). If the incident light beam is circularly polarized, equal amounts of energy are absorbed from the left and right circularly polarized components with the consequence that no circular dichroism is observed. When, however, the medium is placed in a magnetic field which is oriented so that the direction of the magnetic field is the same as that of the propagation direction of the beam of circularly polarized light, the medium will exhibit a selective response to the right and left circularly polarized components.

The contribution of an isolated transition $a \rightarrow j$ to the magnetic circular dichroism of a molecule can be expressed ${ }^{25}$ as the sum of three terms which 
are due to three electronic situations that may be present

$$
[\theta]_{\mathrm{M}}=-21.3458\left\{\mathrm{f}_{1} A+\mathrm{f}_{2}[B+C / k T]\right\}
$$

where $[\theta]_{M}$ is the molar ellipticity per unit magnetic field (deg. decilitres decimetres ${ }^{-1}$ mole $^{-1}$ gauss $^{-1}$ ) and $f_{1}$ and $f_{2}$ are functions (of the resonance frequency $v_{0}$, the frequency of the incident light beam $v$, and the half-band width $\Gamma$ ) which provide an explicit description of the shapes of the MCD bands associated with the $A$ and with the $B$ and $C$ parameters, respectively.

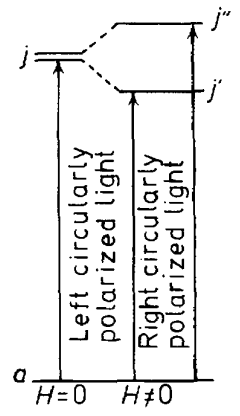

(a) Energy level diagram

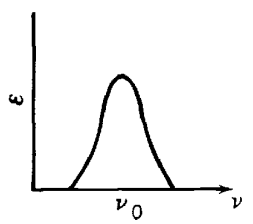

(b) Absorption for $\mathrm{H}=\mathrm{O}$

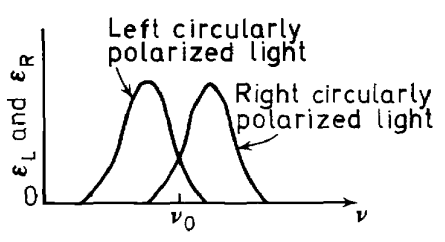

(c) Absorption for $H \neq 0$

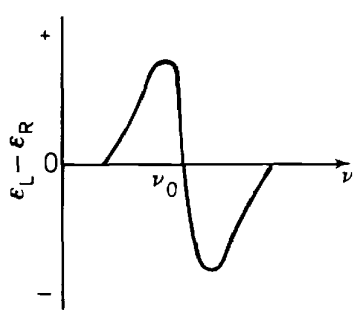

(d) Resultant A term

Figure 2. Illustration of the $A$ term. (a) Energy level diagram for an electronic transition to a degenerate state $j$. (b) Absorption in the absence of a magnetic field. (c) Absorption of left and right circularly polarized light in a magnetic field. (d) Resultant $A$ term MCD curve.

The quantum mechanical expression ${ }^{27}$ for the $A$ term is

$$
A(a \rightarrow j)=\left(1 / 2 d_{a}\right) \Sigma[\langle j|\boldsymbol{\mu}| j\rangle-\langle a|\boldsymbol{\mu}| a\rangle] \cdot \operatorname{Im}\{\langle a|\mathbf{m}| j\rangle \times\langle j|\mathbf{m}| a\rangle\}
$$

where $d_{a}$ is the degeneracy of $a$, and $\boldsymbol{\mu}$ and $\mathbf{m}$ are the magnetic and electric dipole operators, respectively. The basic requirement for a molecule to exhibit an $A$ term is that there must be a non-zero magnetic moment in either the ground state $a$ or in the excited state $j$. This in turn requires that one or more of these states must degenerate. The symmetry requirement for such degeneracies is that the molecule must possess at least a threefold symmetry axis. Consequently, the number of molecules which exhibit $A$ terms will be very small. The origin of the S-shaped bands [Figure 2(d)] which are characteristic of $A$ terms is diagrammatically represented in Figure 2 where we consider the case in which only the state $j$ is degenerate. In the presence of the magnetic field the degeneracy is lifted with the result 
that transitions to the split-levels, $j^{\prime}$ and $j^{\prime \prime}$, now occur at frequencies which are symmetrically located about the zero-field resonance frequency $v_{0}$. The amount of splitting is determined by the magnetic moment between the two states and by the strength of the magnetic field. The selective response [Figure 2(c)] for the absorption of left and right circularly polarized photons to the split states results in two oppositely signed MCD bands [Figure 2(d)] symmetrically located about $v_{0}$ and of equal magnitude since the two transitions occur with equal probability.
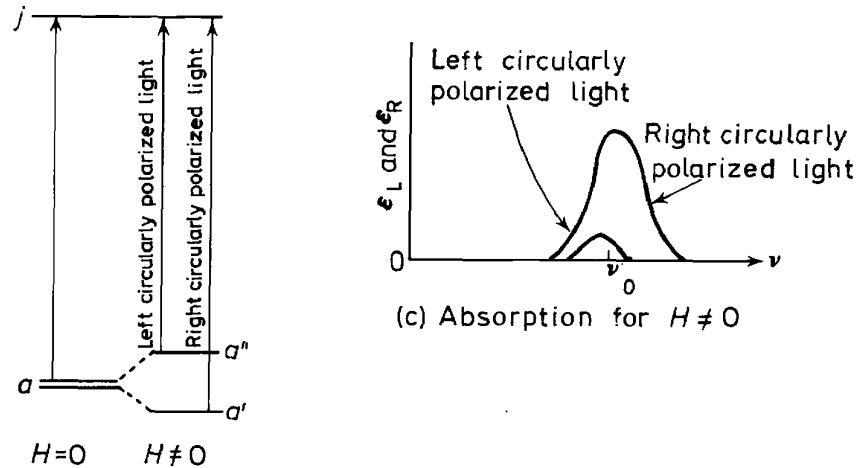

(c) Absorption for $H \neq O$

(a) Energy level diagram

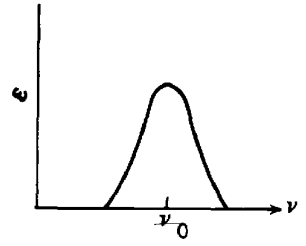

(b) Absorption for $\mathrm{H}=\mathrm{O}$

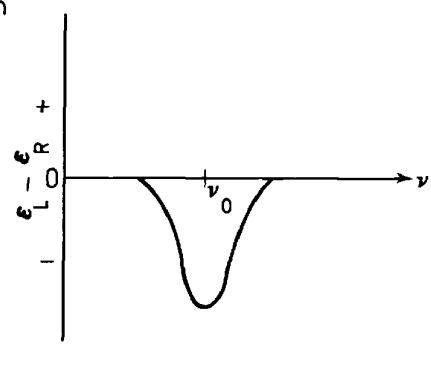

(d) Resultant $C$ term

Figure 3. Illustration of the $C$ term. (a) Energy level diagram for an electronic transition from a degenerate ground state $a$ to a non-degenerate state $j$. (b) Absorption in the absence of a magnetic field. (c) Absorption of left and right circularly polarized light in a magnetic field. (d)

Resultant $C$ term MCD curve.

Figure 3 illustrates the effect of a magnetic field on a molecule for which the ground state $a$ is degenerate. In this situation the lower state $a^{\prime}$ is more highly populated than the upper state $a^{\prime \prime}$. This unequal population, which is even more pronounced at low temperatures, is reflected by the relative intensities of absorption of the left and right circularly polarized components as depicted in Figure 3(c). The resulting experimentally observed $C$ term is shown in Figure 3(d). Such $C$ terms, which have been referred to in the past as 'paramagnetic rotational effects', are very rare among organic compounds (one example is in the negative ions of magnesium phthalocyanine ${ }^{28}$ ), but are more frequent in certain inorganic molecules. Typical examples and applications in the inorganic chemical field have been described elsewhere ${ }^{25}$. 
As indicated in equation $1, C$ terms are described by the same shape function, $\mathrm{f}_{2}$, as are the very common $B$ terms. Since $B$ terms are not subject to temperature dependences, such a test can be employed to differentiate them from $C$ terms.

The low symmetry of the vast majority of organic molecules necessarily precludes the existence of degeneracies in their ground and excited states. Nevertheless, the magnetic field does have an effect on the energy levels of the molecule. The effect is, in fact, a very general one which will be present even in molecules which show $A$ and $C$ terms. This ubiquitous Faraday parameter, the $B$ term, arises from the mixing of the ground and two or more excited states by the magnetic field.

The quantum mechanical expression ${ }^{27}$ for the $B$ term is

$$
\begin{array}{r}
B(a \rightarrow j)=\frac{1}{d_{a}} \sum \operatorname{Im}\left\{\sum_{k \neq a} \frac{\langle k|\boldsymbol{\mu}| a\rangle \cdot\langle a|\mathbf{m}| j\rangle \times\langle j|\mathbf{m}| k\rangle}{h v_{k a}}\right. \\
\left.+\sum_{k \neq j} \frac{\langle j|\boldsymbol{\mu}| k\rangle \cdot\langle a|\mathbf{m}| j\rangle \times\langle k|\mathbf{m}| a\rangle}{h v_{k j}}\right\}
\end{array}
$$

where $h v_{k_{a}}=E_{k}-E_{a}$ and $h v_{k_{j}}=E_{k}-E_{j}$ and $E_{a}, E_{j}$ and $E_{k}$ are the energies of states $a, j$ and $k$, respectively. In order to simplify this discussion we assume that the dominant contribution to the $B$ term comes from the second summation in equation 3. Furthermore, we consider that only three states are involved, namely a ground state $a$ and two excited states $j$ and $k$ as represented diagrammatically in Figure 4(a). In the absence of a magnetic field the promotion of an electron to states $j$ and $k$ is independent of the state of polarization of the incident light - provided that the molecule is not optically active- and absorption is observed at the resonance frequencies $v_{j a}$ and $v_{k a}$ as depicted in Figure 4(b).

If, however, there are off-diagonal matrix elements in the magnetic moment between the states $j$ and $k$, then these states are mixed by the magnetic field. That is, the magnetic field causes each state to aquire some of the characteristics of the other. Now there will be differential absorption of the left and right circularly polarized components as shown in Figure 4(c). This differential absorption results in MCD bands which, for allowed transitions, pass through their maximum values at frequencies corresponding to the zero-field resonance frequencies of the transitions $v_{j a}$ and $v_{k a}$ as shown in Figure 4(d). The preferential absorption of the left and right circularly polarized components will, however, be different for the two transitions [Figure 4(c)]. Consequently, if a positive MCD band is observed at $v_{k a}$, then a negative band will be observed at $v_{j a}$.

In the second summation in the expression for the $B$ term 3 it will be noted that the numerator involves the product of three vectors, namely: the magnetic moment between the states $j$ and $k$ and the electric dipole moments between the ground state $a$ and the two excited states, whereas the denominator contains the energy difference between the states $j$ and $k$. Consequently, in this idealized situation, the two oppositely signed bands will have the same integrated intensity irrespective of the strength of the absorption band. The magnitudes of the two MCD bands depend, however, 


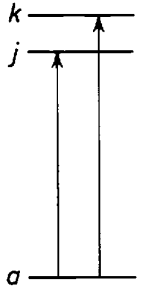

(a) Energy level diagram

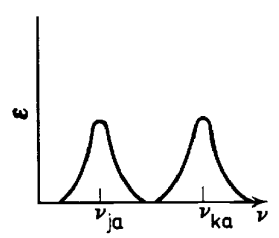

(b) Absorption for $\mathrm{H}=\mathrm{O}$

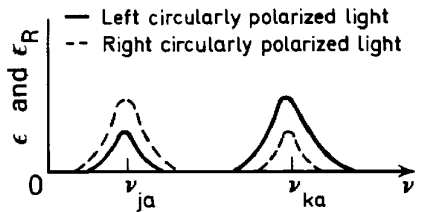

(c) Absorption for $H \neq O$

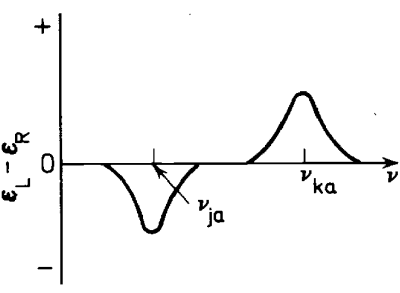

(d) Resultant $B$ terms

Figure 4. Illustration of the $B$ term. (a) Energy level diagram for electronic transitions to two non-degenerate states $j$ and $k$. (b) Absorption in the absence of a magnetic field. (c) Absorption of left and right circularly polarized light in a magnetic field. (d) Resultant $B$ term MCD curves.

on the energy separation between the two excited states. As the energy separation between the two transitions becomes smaller, the intensities of the MCD bands will increase. When the two absorption bands are not resolved, the MCD curve will closely resemble the S-shape characteristic of an $A$ term. Finally, it should be noted that the numerator is in the form of a scalar triple product. Consequently, the MCD bands will be largest when the transition moments are orthogonally oriented with respect to each other. As the angle between any two, say the electric dipole moments, decreases, the MCD bands will become smaller and in the event that any two of the transition moments are parallel no MCD will be observed.

\section{SOME ORGANIC CHEMICAL APPLICATIONS}

Faraday effect studies on compounds which contain either the porphyrin skeleton or else a closely related one have been of particular interest to both experimental ${ }^{19,29-37}$ and theoretical chemists ${ }^{27,28,37,38}$. As a specific example, the MCD and absorption spectra of $\mathrm{Mg}(\mathrm{II})$ deuteroporphyrin IX dimethyl ester are shown in Figure 5. The general features observed in the absorption spectrum are common to all metalloporphyrins; however, the positions and intensities of the three prominent absorption bands vary somewhat with the particular metal incorporated and the nature and pattern of the side chain substitution ${ }^{39}$. The intense absorption band at $408 \mathrm{~nm}$ is the familiar Soret band. The absorption band at $580 \mathrm{~nm}$ is commonly 
designated as the $Q_{0}$ band and the one at $543 \mathrm{~nm}$ is a vibrational overtone of the $Q_{0}$ band. Notwithstanding the dissymmetry in the nature and pattern of the side chain substitution, the very characteristic $A$ terms associated with these absorption bands provides convincing evidence that the effective electronic symmetry of $D_{4 h}$ is obtained and that the three bands are associated with transitions to degenerate excited states which have ${ }^{1} E_{u}$ symmetry ${ }^{27}$.

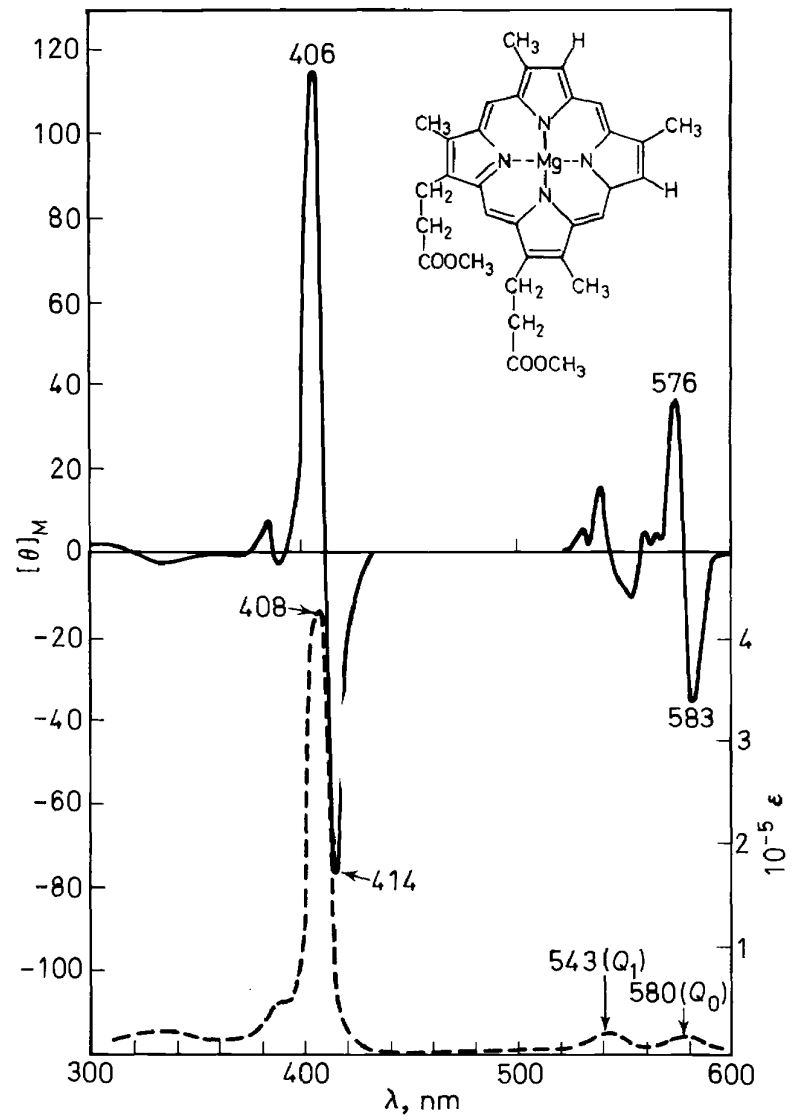

Figure 5. MCD (--_-) and absorption (---) spectra of $\mathbf{M g}$ (II) deuteroporphyrin IX dimethyl ester in benzene. $[\theta]_{M}$ is normalized to 1 gauss.

The magnetic circular dichroism spectra of metalloporphyrins and phthalocyanines have been of some interest to theoretical chemists in recent years since magnetic circular dichroism provides a very direct means of experimentally measuring excited state magnetic moments. The values so obtained can then be compared with the calculated values obtained using the wavefunctions from various theoretical models in order to determine their validity. An interesting result ${ }^{27}$ is that the free-electron theory developed twenty years ago by W. T. Simpson ${ }^{40}$ provides nearly the same ratio of splitting in the Soret and $Q$ bands as do the more sophisticated configuration interaction models developed more recently by Gouterman et $a l^{41}$. 
In the past year we have been interested in a very practical aspect of the MCD of metalloporphyrins. It will be noted in expression 2 for the $A$ term that its magnitude depends on the scalar triple product of the magnetic moment between the split energy levels and on the electric dipole moments between the ground state and the two upper levels. In addition the shape function, $f_{1}$, indicates that the magnitude will be inversely proportional to the half-band width $(\Gamma)$. Consequently, since the absorption bands of metalloporphyrins are very narrow and intense, one would expect that the $A$ terms exhibited by these compounds would be quite large. In fact, as little as $0.000007 \mathrm{mg} / \mathrm{ml}$ of $\mathrm{Mg}$ (II) deuteroporphyrin IX dimethyl ester can readily be detected with our instrument. This sensitivity as well as the characteristic S-shaped bands in two regions of the spectrum make magnetic circular dichroism a uniquely useful analytical spectroscopic method for the detection of very small amounts of these substances. Our interest in this analytical application was based on the idea that since metalloporphyrins have been detected in meteorites these substances are likely to be present on the surface of the moon. Consequently, the MCD spectrum of a portion of the organic extracts from a lunar soil sample was measured ${ }^{35}$. The conclusion reached was that metalloporphyrins are not present in amounts greater than 0.000007 $\mathrm{mg} / \mathrm{ml}$. This result was not entirely unexpected since other analytical techniques indicated that the total organic content in the lunar samples was of the order of one part per million and the extract measured was from a $12.5 \mathrm{~g}$ soil sample.

Although some preliminary MCD measurements have been made on iron porphyrins ${ }^{19},{ }^{37}$, the principal applications of the Faraday effect to the study of these compounds has so far been by MOR. In particular, Volkenstein $^{32}$ and Shashoua ${ }^{42}$ have reported MOR spectra for haemoglobin and oxyhaemoglobin. Shashoua has noted that the natural optical rotations of a number of ferrohaemoglobins ${ }^{42}$ having $\mathrm{H}_{2} \mathrm{O}, \mathrm{O}_{2}$ and $\mathrm{CO}$ as ligands are quite small in the $450-650 \mathrm{~nm}$ region whereas substantial changes can be noted in the MOR spectra. Similar differences were noted in the MOR spectra of cytochrome $\mathrm{c}$ as a function of its oxidation state. In a later publication ${ }^{31}$ Shashoua used the great differences in the MOR spectra of the oxidized and reduced forms of this compound in order to study the kinetics of the redox reaction.

The MOR spectra of haemoglobin and oxyhaemoglobin (modified from Volkenstein ${ }^{32}$ to conform with the sign convention in current use ${ }^{24,25}$ ) and the corresponding MCD spectra measured in our laboratory are shown in Figures 6 and 7 in order to illustrate the differences in the curves obtained by MOR and by MCD. In Figure 6 the MOR $A$ term associated with the $576 \mathrm{~nm}$ absorption band of oxyhaemoglobin is characterized by the intense positive peak at $576 \mathrm{~nm}$ and the weaker negative lobes at 593 and $559 \mathrm{~nm}$. The corresponding MCD $A$ term (Figure 7) is characterized by an S-shaped band (negative maximum at $582 \mathrm{~nm}$; positive maximum at $570 \mathrm{~nm}$ ). Comparison of the curves in Figures 6 and 7 shows that the $A$ term associated with the $414 \mathrm{~nm}$ absorption band of oxyhaemoglobin is much more evident in the MCD spectrum (negative maximum at $425 \mathrm{~nm}$; positive maximum at $408 \mathrm{~nm}$ in Figure 7) than it is in the MOR spectrum (positive maximum at $417 \mathrm{~nm}$ in Figure 6). The CD spectrum of oxyhaemoglobin is included in 

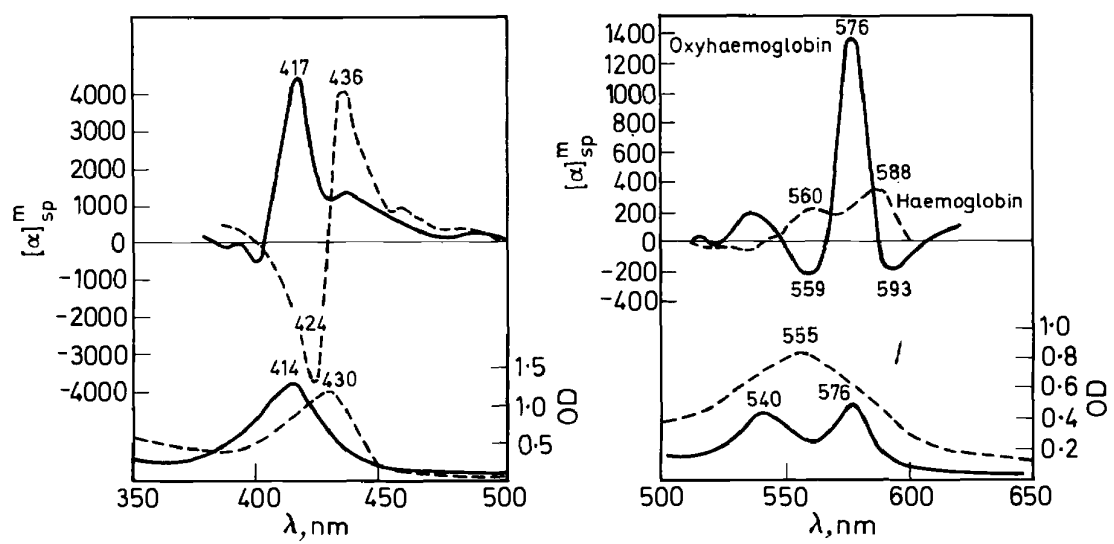

Figure 6. MOR and absorption spectra of horse haemoglobin (-- - ) and oxyhaemoglobin

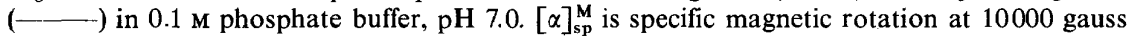
(modified from ref. 32.)

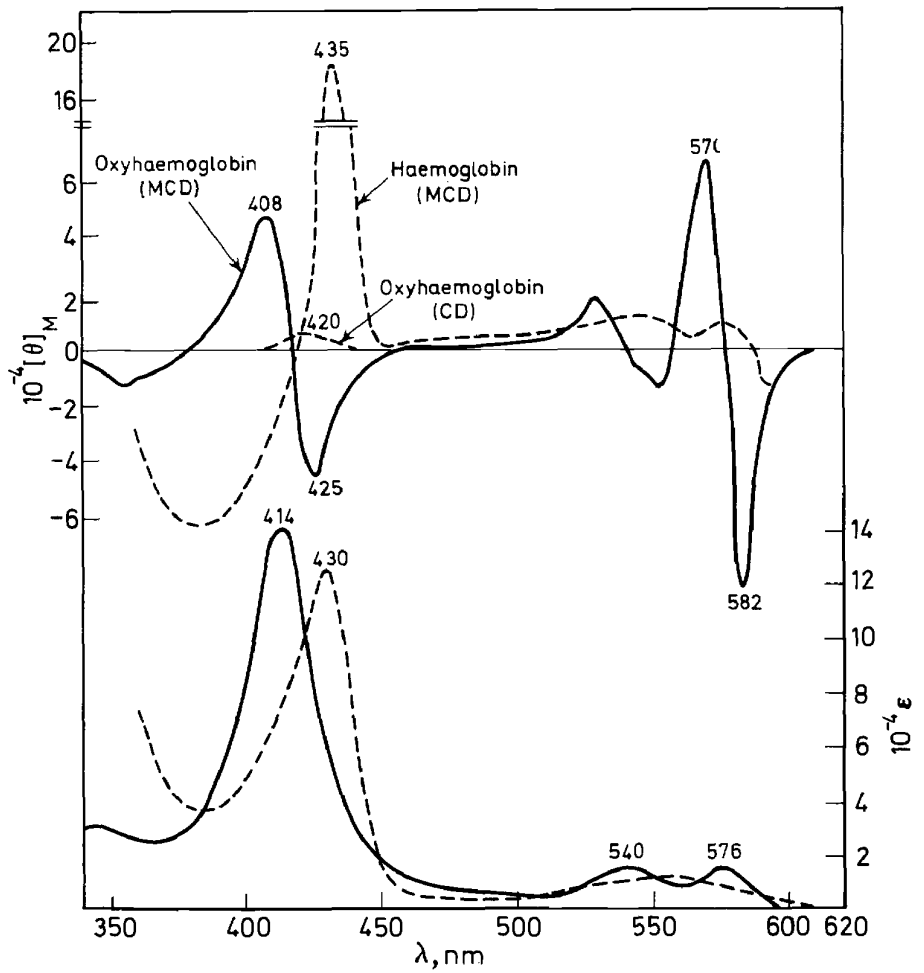

Figure 7. MCD and absorption spectra of human haemoglobin (via sodium dithionite) (---): MCD (-), absorption (- $\longrightarrow$ ) and CD (---) spectra of oxyhaemoglobin in $0.1 \mathrm{M}$ phosphate buffer, $\mathrm{pH}$ 7.0. $[\theta]_{\mathrm{M}}$ is reported for a 49600 gauss magnetic field. 
Figure 7 to illustrate the great differences in the MCD and CD signals. Only the CD Cotton effect at about $420 \mathrm{~nm}$ could be measured at the concentration $\left(4 \times 10^{-6}\right.$ molar) used for the MCD measurements. The exchange of the ligand $\mathrm{O}_{2}$ in oxyhaemoglobin for $\mathrm{H}_{2} \mathrm{O}$ in haemoglobin results in dramatic changes in the MOR (Figure 6) and MCD (Figure 7) spectra. The shape of the MCD curve of a haemoglobin (Figure 7) suggests a significant contribution of a $B$ term in the $430 \mathrm{~nm}$ region. In the MOR spectrum (Figure 6) this $B$ term has a characteristic S-shape (positive maximum at $436 \mathrm{~nm}$; negative maximum at $424 \mathrm{~nm}$ ).

The MCD spectrum of a metalloporphyrin, $\mathrm{Mg}$ (II) deuteroporphyrin IX dimethyl ester, was used (Figure 5) to illustrate $A$ terms. The MCD spectrum of protoporphyrin IX is shown in Figure 8 as an example of a structurally related compound which exhibits only $B$ terms. The effect of removing the metal and protonating the two opposite nitrogen atoms of the porphyrin is to cause electronic displacements in the $x$ and $y$ directions in the plane of the molecule to become strongly unequivalent, i.e. the excited states are no longer degenerate. This splitting is reflected in the absorption spectrum by the four bands at 630,576, 541 and $505 \mathrm{~nm}$ which are derived from the metalloporphyrin $Q_{0}$ and $Q_{1}$ bands (see Figure 5). In the MCD spectrum the negative $B$ terms (but positive $B$ values-see equation 1) at 628 and 582 $\mathrm{nm}$ are associated with the transitions $\left(Q_{0}^{x}\right.$ and $\left.Q_{1}^{x}\right)$ which are polarized in the $x$-direction whereas the positive $B$ terms at 540 and $511 \mathrm{~nm}$ are associated with the corresponding $y$-polarized transitions $\left(Q_{0}^{y}\right.$ and $\left.Q_{1}^{y}\right)$. The nearly equal intensities of the corresponding $B$ terms, e.g. $Q_{0}^{x}$ and $Q_{0}^{y}$, indicate ${ }^{27}$ that intermixing of $Q$ states dominates over mixing with higher states. Although the splitting of the Soret band (at $407 \mathrm{~nm}$ ) is not evident in the absorption spectrum, it is very apparent in the MCD spectrum. In particular, the intense S-shaped MCD band characteristic of an $A$ term (see Figure 5) is noticeably absent in the region of the Soret band of protoporphyrin IX (Figure 8). Instead one observes two negative MCD bands at 426 and $379 \mathrm{~nm}$ and a positive band at $407 \mathrm{~nm}$. The intensities of these bands as well as the shape of the MCD curve in the $350-450 \mathrm{~nm}$ region indicate the presence of several closely spaced $B$ terms.

We have already called attention to the separate physical origins of natural optical activity and magnetic optical activity. Since magnetic optical activity is a universal property of all matter irrespective of whether or not the sample is itself optically active, it is of some interest to compare and contrast in some optically active substances the type of information provided by MCD with that provided by $\mathrm{CD}$. As a particular example ${ }^{33}$, the absorption, MCD and CD spectra of isochlorin- $e_{5}$ dimethyl ester is shown in Figure 9. The chlorins are derived from the porphyrins by reduction of one of the pyrrole ring double bonds. The very intense Soret band is found at $400 \mathrm{~nm}$. The very weak band at $527 \mathrm{~nm}$ is designated $Q_{0}^{x}$ and the much stronger one at $661 \mathrm{~nm}$ is designated $Q_{0}^{y}$. The bands on the blue sides of these two bands are the respective overtones. The same fundamental picture is obtained in the MCD spectrum as in the metal-free porphyrins, namely, that the MCD bands associated with the $Q_{0}^{y}$ and $Q_{0}^{x}$ bands are of opposite sign. However, it will be noted that the relative signs of these two bands are opposite to that found in the metal-free porphyrins (Figure 8); i.e. the long wavelength band 
is now positive. In the Soret region the splitting of this transition is again dramatically revealed in the magnetic circular dichroism spectrum but much less so in the absorption spectrum.

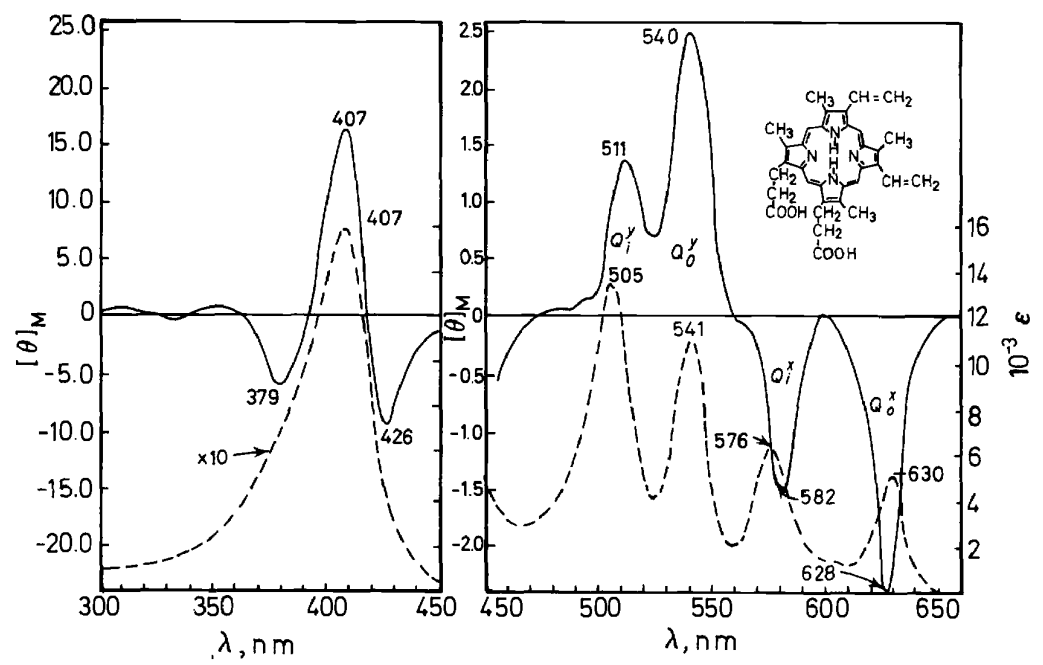

Figure 8. MCD (- $)$ and absorption (- - - ) spectra of protoporphyrin IX in $\mathrm{CHCl}_{3}$. $[\theta]_{\mathbf{M}}$ is normalized to 1 gauss.

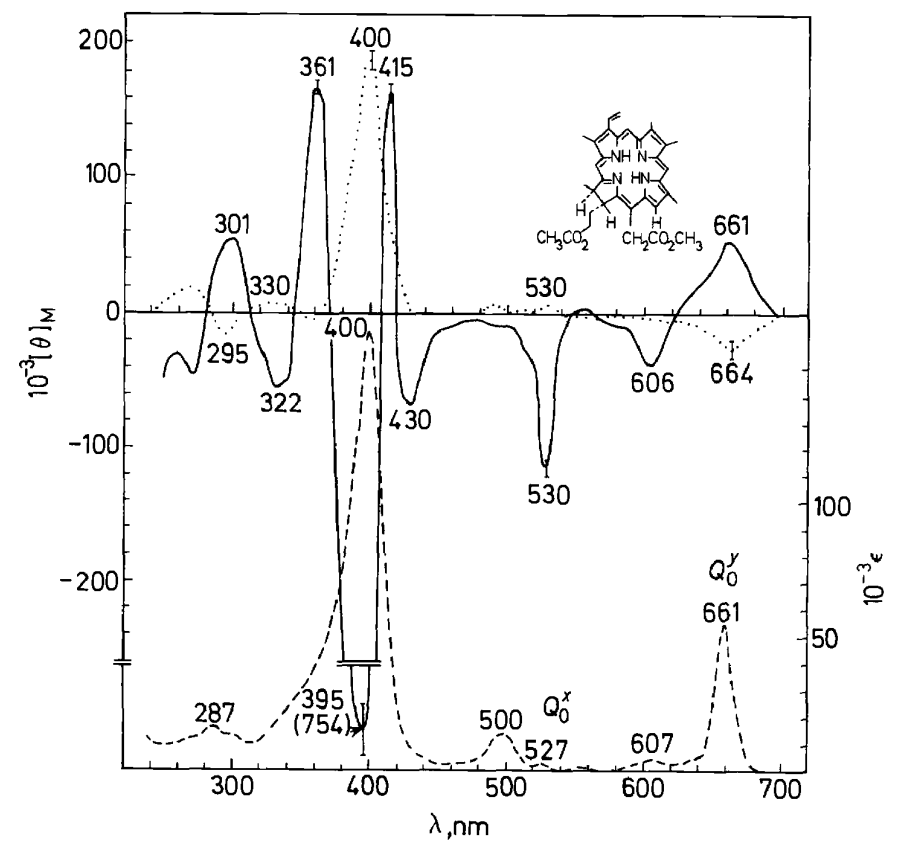

Figure 9. $\mathrm{MCD}(--), \mathrm{CD}(\ldots)$ and absorption (-- ) spectra of isochlorin- $\mathrm{e}_{5}$ dimethyl ester in dioxane. $[\theta]_{M}$ is reported for a 41700 gauss magnetic field. 
In this investigation ${ }^{33}$ we compared the $\mathrm{MCD}$, absorption and $\mathrm{CD}$ spectra of six closely related optically active chlorins. The results of the analysis indicated that, if MCD instruments were generally available, this would be the preferred method for correlating spectra with the nature and positions of substituents on the chlorin skeleton. The principal reason is that the signals are very much stronger and consequently easier to measure accurately than in $\mathrm{CD}$ or absorption. Furthermore, the magnitude of one of the MCD bands, in particular the one at $530 \mathrm{~nm}$ which is associated with the $Q_{0}^{x}$ transition, is very sensitive to both the nature and the positions of the substituents on the chlorin framework. For example, the MCD, CD and absorption spectra of chlorin-e $\mathrm{e}_{6}$ trimethyl ester are shown in Figure 10, where it will be noted that the effect of replacing the hydrogen atom with the carbomethoxy group is to intensify greatly the $534 \mathrm{~nm}$ MCD band. The curves in Figures 9 and 10 also illustrate the fact that there is no direct relationship between the intensities in the absorption and in the MCD spectrum. In particular, in Figure 10 compare the intensities of the MCD bands associated with the absorption bands at 530 and at $665 \mathrm{~nm}$.

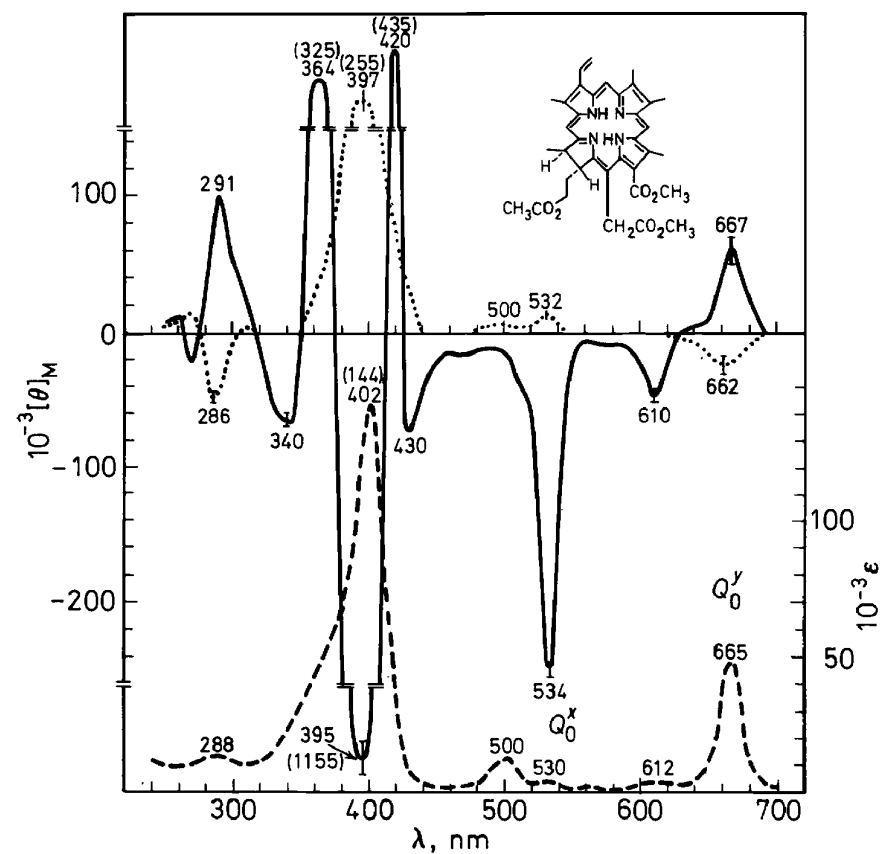

Figure 10. MCD (- - , CD (...) and absorption (-- - ) spectra of chlorin- $\mathrm{e}_{6}$ trimethyl ester in dioxane. $[\theta]_{M}$ is reported for a 41700 gauss magnetic field.

In a recent investigation Houssier and Sauer ${ }^{34}$ made an extensive study of the CD and MCD spectra of several chlorophyll and protochlorophyll pigments and their phaeophytins. These authors made effective use of MCD and $\mathrm{CD}$ as complementary spectroscopic techniques for absorption band assignments. 
As part of another investigation ${ }^{36}$ we examined the MCD spectra of a series of corrin derivatives which differed in the substituents at tached to the corrin skeleton and in the particular metal $(\mathrm{Co}, \mathrm{Ni}, \mathrm{Zn})$ coordinated with the four nitrogen atoms of the reduced pyrrole rings. The MCD and absorption spectra of one member of this series are shown in Figure 11 to illustrate the application of MCD to the detection of hidden absorption bands among corrins. In particular, attention should be called to the $330-450 \mathrm{~nm}$ region, which displays much more detail in its MCD curve as compared to its absorption spectrum.

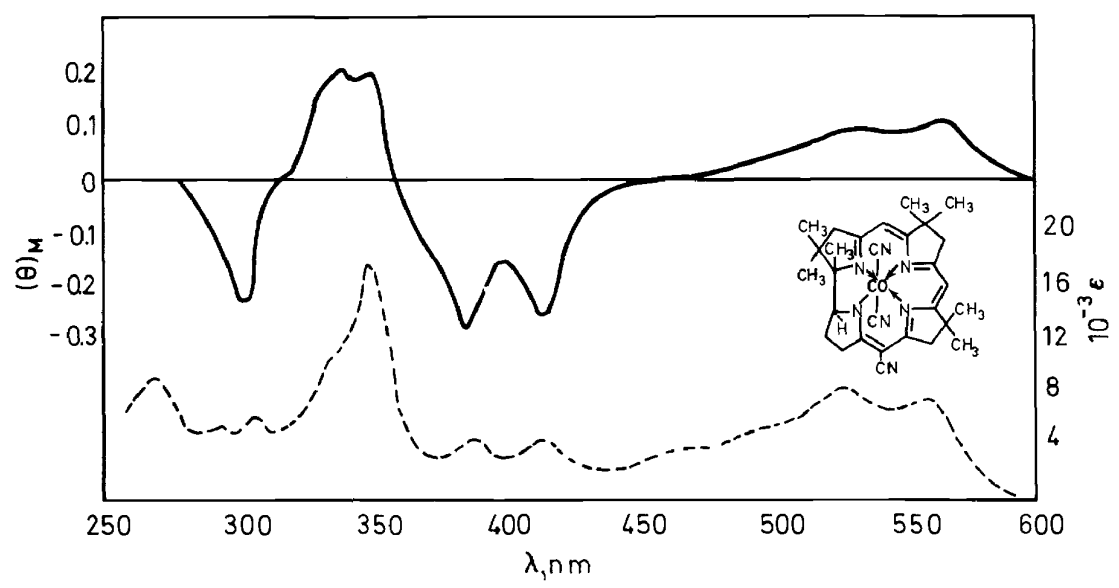

Figure 11. MCD (- - ) and absorption (-- - ) spectra of racemic dicyanocobalt(III).

$1,2,2,7,7,12,12$-heptamethyl-15-cyanocorrin in ethanol. $[\theta]_{M}$ is normalized to 1 gauss.

Aromatic hydrocarbons have provided a fertile series for investigation by means of Faraday effect spectroscopy during the last decade. The application $^{43,44}$ of MCD as an aid to the assignment of electronic transitions in the case of coronene and triphenylene (and therefore, presumably for benzene as well) has been summarized in the review of Schatz and McCaffery ${ }^{25}$.

We have investigated the MCD spectra of a number of annulene derivatives $^{45}$. Figure 12 shows the absorption and MCD spectra of a particularly important member of this series, namely [18] annulene. This molecule has been of considerable interest to theoretical chemists in recent years because of the question of aromaticity in the larger cyclic polyenes. Many attempts have been made to explain quantitatively the absorption spectrum of this compound ${ }^{46,47}$. All molecular orbital treatments based on the assumption of no bond alteration failed in that they led to excessively low energies and excessively large oscillator strengths. On the other hand, the hypothesis of bond alternation was not supported by $\mathrm{x}$-ray diffraction measurements ${ }^{48}$. The situation has been recently reviewed by Salem ${ }^{49}$ and a possible explanation provided for the discrepancy between the previously invoked schemes. A molecular distortion is assumed, owing to a slight overcrowding of the internal hydrogens. This tends to lengthen the outer $\mathrm{C}-\mathrm{C}$ bonds with respect to the inner ones. According to this explanation, [18] annulene should 
keep its centre of symmetry and consequently its $D_{6 h}$ symmetry. The MCD spectrum of [18] annulene (Figure 12) is consistent with this explanation since the observation of an $A$ term corresponding to the $368 \mathrm{~nm}$ absorption band confirms the assignment ${ }^{46}$ of this band to the ${ }^{1} E_{1 u}$ degenerate transition. It is of particular interest to note that the sign of the $A$ term in [18] annulene is opposite to that in $\mathrm{Mg}$ (II) deuteroporphyrin IX dimethyl ester (Figure 5). The weaker absorption band at $448 \mathrm{~nm}$ is probably due to the electronically forbidden but vibrationally allowed $B_{1 u}$ transition. The $B_{2 u}$ transition presumably underlies the absorption shoulders at longer wavelengths. The MCD bands associated with these transitions are quite small. Other interesting examples were provided ${ }^{45}$ by several derivatives of [18] annulene in which pairs of central hydrogen atoms were replaced by bridges containing various combinations of sulphur and oxygen. For example, in [18] annulene trioxide the sign of the $A$ term is reversed as compared to the unbridged compound. In the case of the trisulphide derivative the very much smaller effects were interpreted as reflecting a reduction in the symmetry of the framework through overcrowding of the inner atoms and consequent twisting of the molecule.

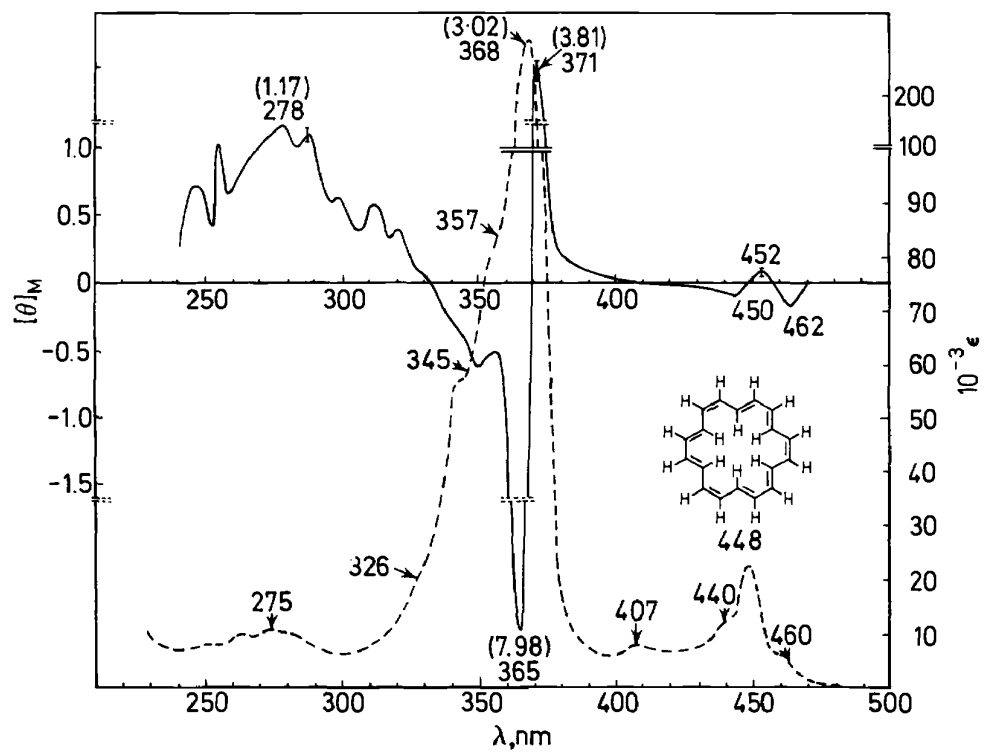

Figure 12. MCD (-) and absorption (-- ) spectra of [18] annulene in isooctane. $[\theta]_{M}$ is normalized to 1 gauss.

In the introductory section the $B$ term was illustrated in Figure 4 with the idealized three state case. Since the expression for the $B$ term requires the summation over all excited states, the simple features illustrated are rarely encountered in practice. The information provided in such cases is, however, not without interest. This is particularly true when a closely related series of compounds is available for study. Thus an extension of our annulene studies ${ }^{45}$ to a series of 1,6 bridged [10]-annulenes ${ }^{50}$ revealed that the general shape, 
sign and magnitude of the $B$ terms through the forbidden ${ }^{1} L_{b}$ band (around $380 \mathrm{~nm}$ ) are very sensitive to the nature of the substituent on the 1,6-bridge, whereas only intensity changes were noted in the absorption spectra. As a specific example, the MCD spectra of 1,6-iminocyclodecapentaene measured in methanol and in methanolic $\mathrm{HCl}$ are compared in Figure 13. The protonation of this compound causes drastic changes in the MCD spectrum (an almost identical curve resulted when boron trifluoride etherate was added), which suggests a strong interaction between the nonbonding nitrogen electrons and the ten- $\pi$-electron system of the ring.
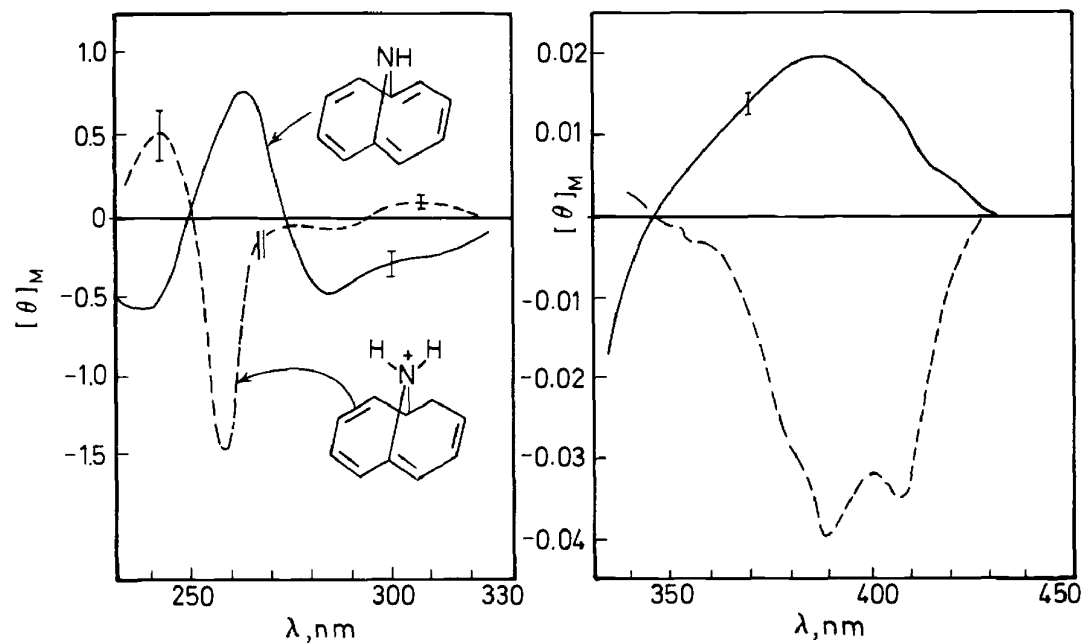

Figure 13. MCD spectra of 1,6-iminocyclodecapentaene in methanol (-_-) and in methanolic $\mathrm{HCl}(---) \cdot[\theta]_{\mathrm{M}}$ is normalized to 1 gauss.

Additional examples can be provided from aromatic compounds. Thus, in a preliminary investigation of the MCD spectra of aromatic systems we $\mathrm{e}^{21}$ called attention to the sensitivity of MCD to steric strain in some derivatives of benzo(c)phenanthrene. Foss and McCarville ${ }^{51}$ compared the MCD spectra of several catacondensed aromatic hydrocarbons (e.g. naphthalene, anthracene, etc.) and suggested that the changes in the sign of the MCD in a homogeneous series could be used as a means of labelling electronic transitions.

In a subsequent investigation, Foss and McCarville ${ }^{52}$ measured the MCD of a series of substituted benzenes and found a correlation between the electron donating or withdrawing power of the substituent and the sign as well as of the intensity of the $B_{2 u}$ band. In particular, it was found that a linear relation existed between the Hammett $\sigma_{\text {para }}$ value of the substituent and the MCD for both mono- and di-substituted benzenes. In addition, these authors noted that the symmetry of the molecule appeared to play an important role in determining the magnitude of the MCD. It is anticipated that the results of a theoretical study in progress ${ }^{53}$ will provide a firm basis for understanding these substituent effects. 
CARL DJERASSI, EDWARD BUNNENBERG AND DANNY ELDER

Relatively little work has been done on simple nitrogen bases and the reports which have been published on the $\mathrm{MOR}^{17}$ and $\mathrm{MCD}^{54}$ spectra of these compounds have largely been limited to exploratory studies. It has been suggested ${ }^{55}$ that MCD may be a very suitable technique for locating $\mathrm{n}-\pi^{*}$ transitions in nitrogen bases since the MCD bands associated with the $\mathrm{n}-\pi^{*}$ and $\pi-\pi^{*}$ transitions were predicted to be of opposite sign. However, this does not appear to be generally the case. A more detailed theoretical study of the MCD of simple nitrogen bases is in progress ${ }^{53}$.

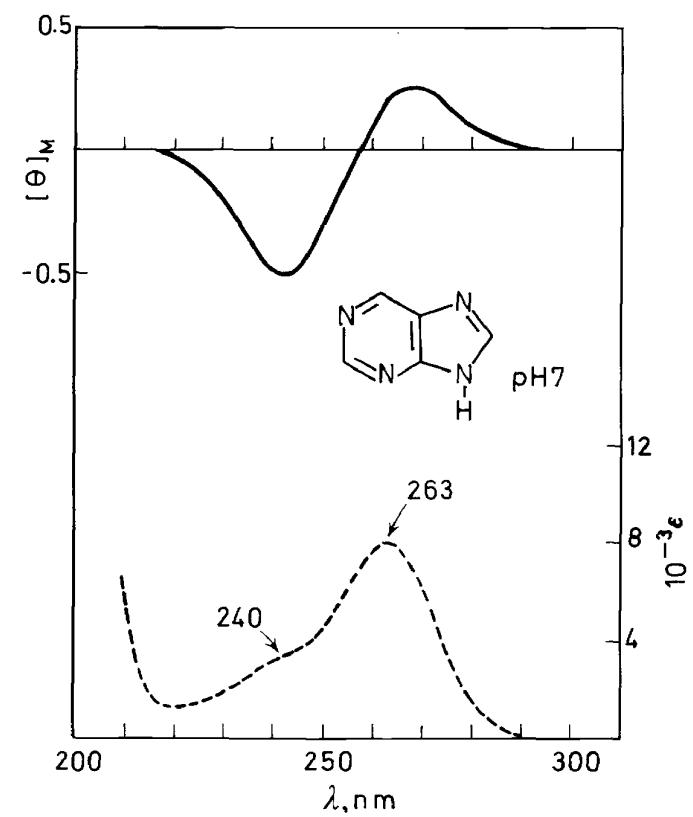

Figure 14. MCD (- $)$ and absorption (-- spectra of purine in water at $\mathrm{pH}$ 7. $[\theta]_{\mathrm{M}}$ is normalized to 1 gauss.

A recent series of papers ${ }^{56-58}$ from our laboratory records the applications of MCD to another class of heterocyclic compounds, the purine and pyrimidine bases and their nucleoside derivatives. Our investigations in this area were primarily motivated by the fact that the purine and pyrimidine nucleosides are constituents of the biologically important nucleic acids and that any concerted investigation of the applications of MCD in such polymeric systems must be based on a detailed preliminary examination of their monomeric chromophores. In addition, we considered that the availability of the component purine and pyrimidine bases as well as several nucleoside anomers afforded the opportunity of comparing and contrasting more closely the kind of information provided by magnetic and natural circular dichroism. Furthermore, it was of interest to us to explore the potential analytical applications of MCD for detecting very small quantities of these compounds.

The MCD and absorption spectrum of purine itself is reproduced in Figure 14. By analogy to the symmetry classification of the absorption bands 
of benzene the strong band at $263 \mathrm{~nm}$ is assigned to the $B_{2 u}$ transition. The weaker $B_{1 u}$ transition can be observed as a shoulder at about $240 \mathrm{~nm}$ and a pair of $E_{1 u}$ bands are located below $200 \mathrm{~nm}$. The n- $\pi^{*}$ transitions are generally hidden under the more intense $\pi-\pi^{*}$ transitions; however, fluorescence measurements placed one of these at about $280 \mathrm{~nm}$. Although the inequality in the intensities of the two oppositely signed MCD bands associated with the $B_{1 u}$ and $B_{2 u}$ transitions might be interpreted ${ }^{56}$ as reflecting the presence of such a transition, it is not discernible as such.

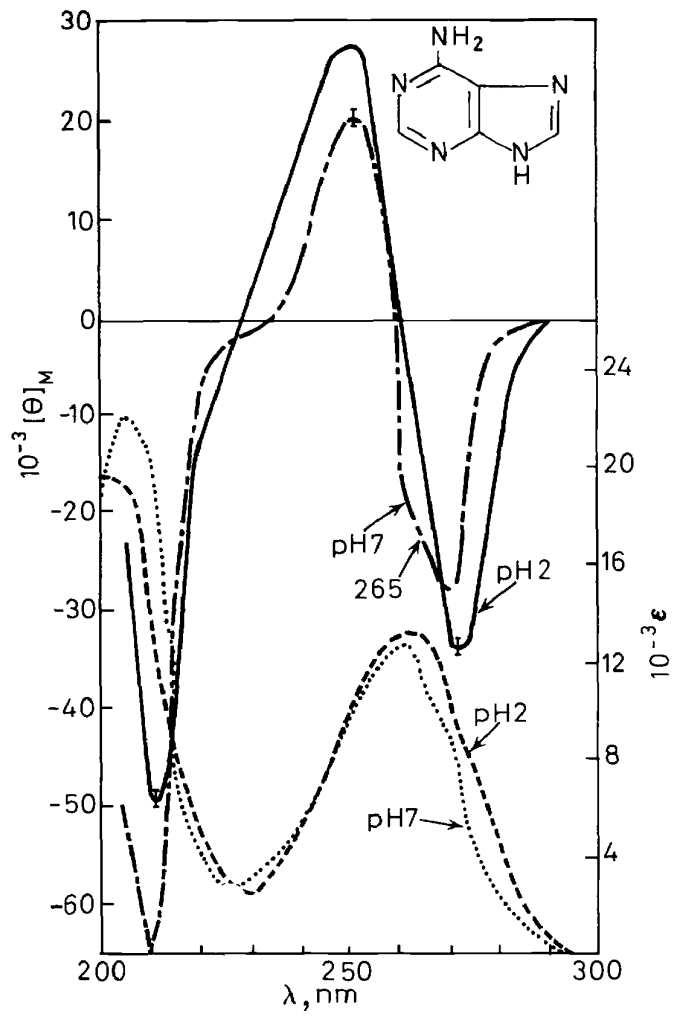

Figure 15. $\mathrm{MCD}$ at $\mathrm{pH} 7(-\ldots)$ and $\mathrm{pH} 2($ ) and absorption at $\mathrm{pH} 7(\ldots)$ and $\mathrm{pH} 2$ $(---)$ of adenine in water. $[\theta]_{\mathrm{M}}$ is reported for a 49500 gauss magnetic field.

An important member of the purine bases which are found in the nucleic acids is adenine. The MCD and absorption spectra of this base measured at pH 7 and at pH 2 are shown in Figure 15 in order to illustrate several points. We have already called attention to the important discovery by Foss and McCarville ${ }^{52}$ of the sign reversal in the $B_{2 u}$ transition of substituted benzenes. This substituent effect was noted by us as well as by McCarville ${ }^{59}$ in the purine series. In particular, it will be noted that the sign of the MCD bands associated with the $B_{2 u}$ and the $B_{1 u}$ transitions in purine (Figure 14) are opposite to those found for adenine (Figure 15). There exists considerably more direct evidence for the presence of an $n-\pi^{*}$ transition in the case of adenine as compared to purine. In particular, we have suggested ${ }^{56}$ that the 
shoulder at $265 \mathrm{~nm}$ in the $\mathrm{pH} 7 \mathrm{MCD}$ spectrum may be due to an $\mathrm{n}-\pi^{*}$ transition since it disappears when the measurement is made in $0.01 \mathrm{~N} \mathrm{HCl}$ and the intensities of the two strong MCD bands become larger. The increased intensities of the two bands could, however, also be attributed to the more nearly orthogonal orientation of the $B_{2 u}$ and $B_{1 u}$ transition moments accompanying protonation or to a decrease in the separation of the energy levels.

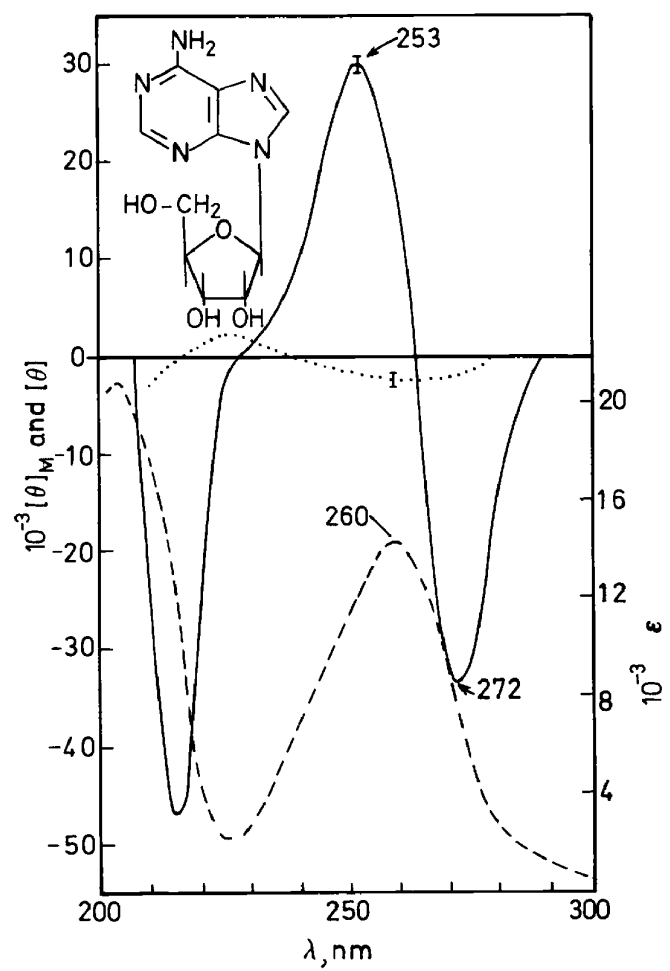

Figure 16. MCD (- $(-, \mathrm{CD}(\cdots)$ and absorption (-- - ) spectra of adenosine in water at $\mathrm{pH}$ 7. $[\theta]_{\mathrm{M}}$ is reported for a 49500 gauss magnetic field.

The MCD, CD and absorption spectra of the corresponding nucleoside, adenosine, are reproduced in Figure 16 in order to illustrate a case in which MCD is of much more value than CD for the purpose of resolving overlapping absorption bands. The oppositely signed MCD bands at $272 \mathrm{~nm}$ and at $253 \mathrm{~nm}$ clearly indicate that the $260 \mathrm{~nm}$ absorption band contains two unresolved components (the $B_{2 u}$ and $B_{1 u}$ transitions). The CD curve of the nucleoside, on the other hand, shows only a weak and poorly defined maximum in this region.

In the pyrimidine bases and nucleosides ${ }^{56}$ the roles of $\mathrm{MCD}$ and $\mathrm{CD}$ are interchanged with respect to the application of the two methods for detecting closely spaced electronic transitions. For example, the observed MCD (i.e. $C D$ plus $M C D$ ), $M C D, C D$ and absorption spectra of the nucleoside uridine 
are shown in Figure 17(a). The absorption spectrum of uridine shows maxima at 261 and at $205 \mathrm{~nm}$. The composite nature of these bands is evident from the CD Cotton effects at 268, 234 and $217 \mathrm{~nm}$ which have been correlated with the $B_{2 u}, B_{1 u}$ and $E_{1 u}$ transitions, respectively ${ }^{60,61}$. The MCD and absorption spectra for the corresponding base, uracil, are presented in Figure 17(b) in order to illustrate a situation occasionally arising in the MCD of optically active compounds. Specifically, the similarity in the shapes of the observed MCD curve and the CD curves of uridine [Figure $17($ a) ] results in a rather poorly defined MCD curve. Consequently, it is desirable in these cases to have the constituent chromophore available for comparison.
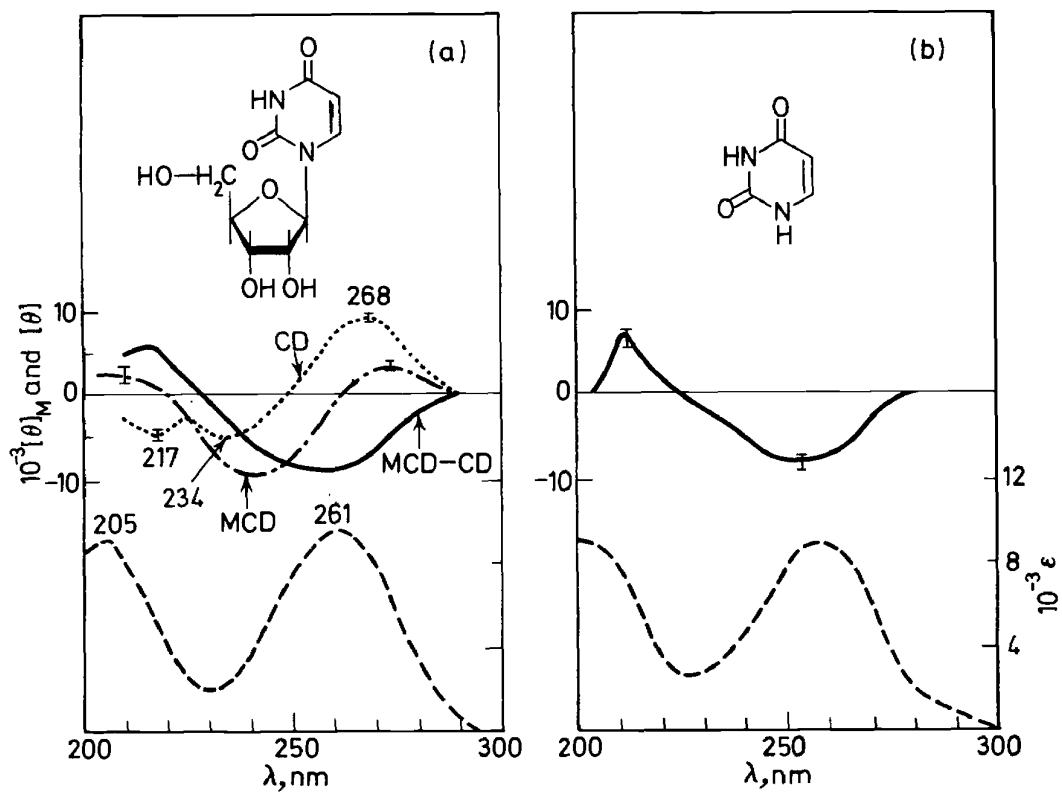

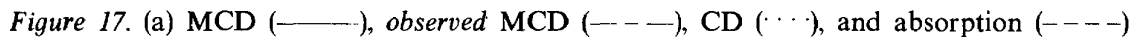
spectra of uridine in water at $\mathrm{pH} 7$. (b) $\mathrm{MCD}\left(-{ }_{-}\right.$and absorption (----) spectra of uracil in water at $\mathrm{pH}$ 7. $[\theta]_{\mathrm{M}}$ is reported for a 49500 gauss magnetic field.

A potentially useful application of MCD is the differentiation between the very small amounts of purine and pyrimidine nucleosides that are frequently isolated in the laboratory. For example, qualitatively the absorption spectra of uridine [Figure 17(a)] and adenosine (Figure 16) are very similar in that both have absorption bands located at nearly the same wavelengths. The MCD spectra of the two nucleosides are, however, very dissimilar. As another specific example we can present the MCD and absorption spectra (Figure 18) of two isomeric purines, namely 1-methyl-6-thiohypoxanthine and 9-methyl-6-thiohypoxanthine. Here it will be noted that the absorption spectra of these compounds are very similar both in terms of positions and intensity of the two absorption bands. Their MCD spectra, however, provide 
a means of differentiation between the two isomers because of the differences below $270 \mathrm{~nm}$.

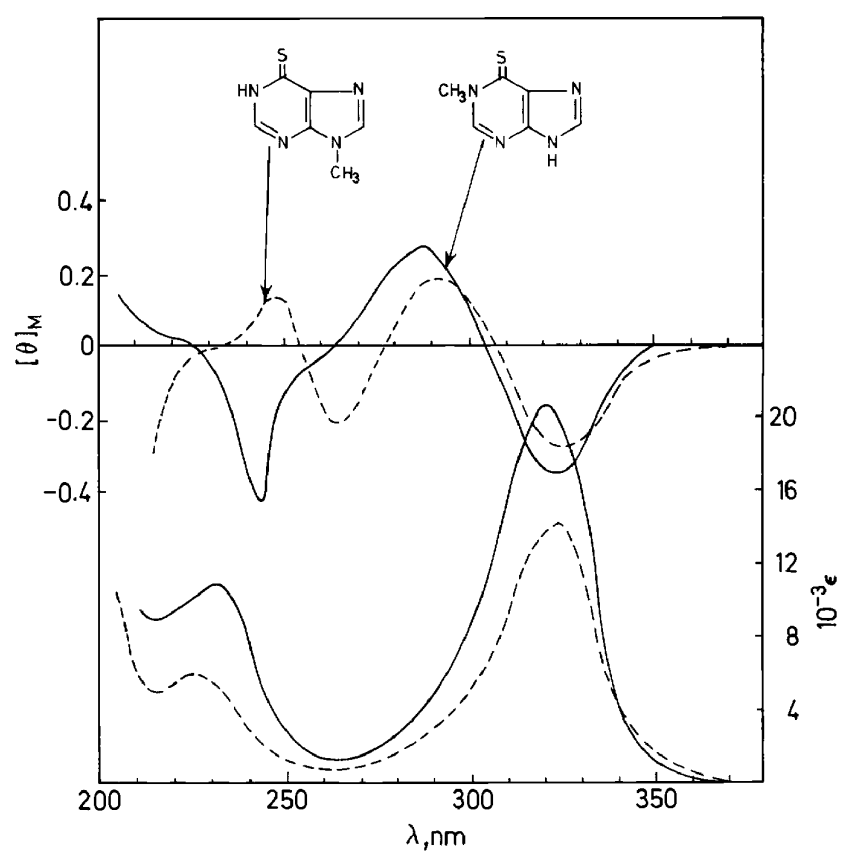

Figure 18. MCD and absorption spectra of 1-methyl-6-thiohypoxanthine (- $\longrightarrow$ and 9-methyl6-thiohypoxanthine (-- - in water at $\mathrm{pH} 2 .[\theta]_{\mathrm{M}}$ is normalized to 1 gauss.

In subsequent studies we investigated the $\mathrm{MCD}$ and $\mathrm{CD}$ spectra of a series of 3,5'-purine cyclonucleosides ${ }^{57}$ and a series of 8-hydroxy and 8 -mercaptoadenosine cyclonucleosides ${ }^{58}$. Particularly in the former study ${ }^{57}$ it was of interest to ascertain whether the generalizations observed in the MCD spectra of ordinary nucleosides ${ }^{56}$ would also be applicable to the cyclonucleosides as well. As a specific example, the MCD, CD and absorption spectra of 2',3'-isopropylidene-3,5'-guanosine cyclonucleoside are reproduced in Figure 19. The MCD spectrum at $\mathrm{pH} 7$ is an example of a situation in which more than two excited states are significantly mixed by the magnetic field. In our analysis we found $\mathrm{CD}$ and MCD to be of mutual and complementary value in the assignments of transitions. The dramatic changes in the MCD and CD spectra in neutral [Figure 19(a)] and in acid solution [Figure 19(b)] can be attributed to an $n-\pi^{*}$ transition and to wavelength shifts in the $\pi-\pi^{*}$ transitions. The shoulder at about $255 \mathrm{~nm}$ in the pH 7 CD spectrum [Figure 19(a)] was taken as evidence ${ }^{60}$ of an $\mathrm{n}-\pi^{*}$ transition whose sign and magnitude obscured the weaker $B_{2 u}$ CD Cotton effect expected in this region. Since on protonation in acid solution $n-\pi^{*}$ transitions are expected to appear at considerably higher energies, the positive Cotton effect associated with the $B_{2 u}$ transition can now be observed at $264 \mathrm{~nm}$ [Figure 19(b)]. The CD Cotton effects at 237 and $202 \mathrm{~nm}$ [Figure 19(b)] are 
correlated with the $B_{1 u}$ and $E_{1 u}$ transitions, respectively. The MCD bands in the $\mathrm{pH} 7$ spectrum [Figure 19(a)] at 268, 248, 233 and $215 \mathrm{~nm}$ were correlated $^{57}$ with the $B_{2 u}, B_{1 u}$ and the $E_{1 u}$ pair transitions, respectively. The unequal intensities of the 268 and $248 \mathrm{~nm}$ MCD bands were considered to be indirect evidence for the presence of an $n-\pi^{*}$ transition at about $260 \mathrm{~nm}$ since in acid solution [Figure $19(\mathrm{~b})]$ the intensities of the $B_{2 u}($ at $270 \mathrm{~nm})$ and the $B_{1 u}$ (at $240 \mathrm{~nm}$ ) bands are nearly equal and much larger as expected if dominant mixing occurs between the two transitions.

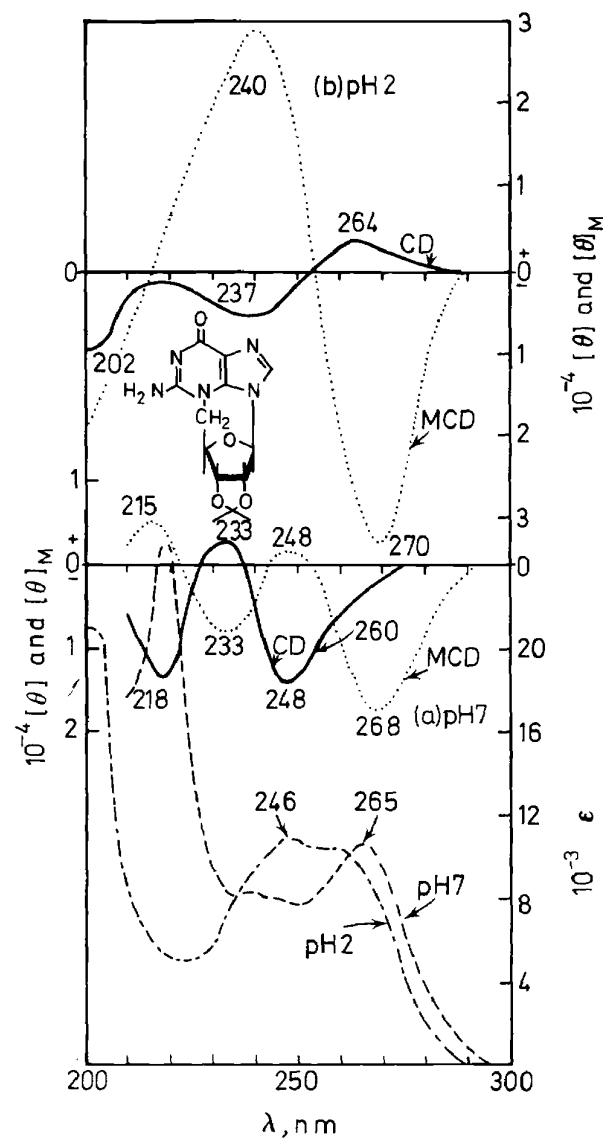

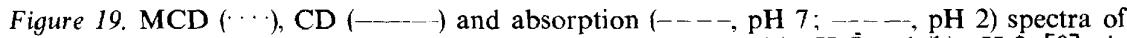
$2^{\prime}, 3^{\prime}$-isopropylidene-3,5'-guanosine cyclonucleoside in water at (a) pH 7 and (b) $\mathrm{pH} 2$. [ $\left.\theta\right]_{\mathrm{M}}$ is reported for a 49500 gauss magnetic field.

Faraday effect studies have also been made on other systems. In particular, Tollin ${ }^{62}$ measured the MCD and CD spectra of various riboflavin analogues and found evidence for the presence of two transitions which had been predicted by molecular orbital calculations ${ }^{63}$ but which had not been detected by ordinary means. In addition, the MCD spectra of the flavin and 
the 6,7-dimethylquinoxaline cations were compared as a means of ascertaining that the position of protonation of the flavins was on the N1 nitrogen<smiles></smiles><smiles>Cc1cc2ncc[nH+]c2cc1C</smiles>

atom of the pyrimidine ring. Marlborough et al. ${ }^{64}$ applied MOR to the study of the oxidized and reduced forms of spinach ferredoxin and Bayer et al. ${ }^{65}$ have investigated the application of MCD and CD for the structure determination of xanthine oxidase.

The carbonyl chromophore has been the subject of a number of MOR $^{16-18,66-68}$ and $\mathrm{MCD}^{69-71}$ investigations. The conflicting nature of the earlier MOR work with respect to theoretical predictions has been noted by Stephens ${ }^{72}$ and the sources of experimental errors in MOR measurements have been discussed by Winkler ${ }^{18,68}$. In fact, reliable MOR ${ }^{18,68}$ and $\mathrm{MCD}^{69-71}$ spectra have been obtained only very recently. The reason for

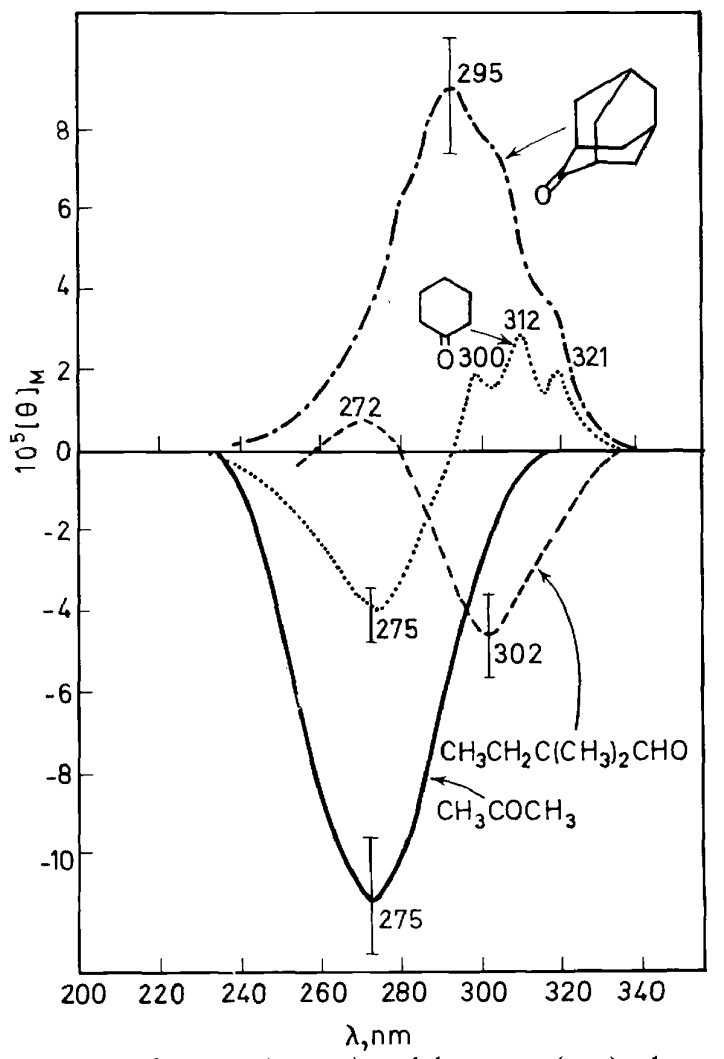

Figure 20. MCD curves of acetone (- - , cyclohexanone ( $\cdots)$, adamantanone $(--\rightarrow)$ and 2,2-dimethylbutanal (-- $)$ in cyclohexane. Vertical bars indicate the signal to noise ratio.

$[\theta]_{\mathrm{M}}$ is normalized to 1 gauss. 


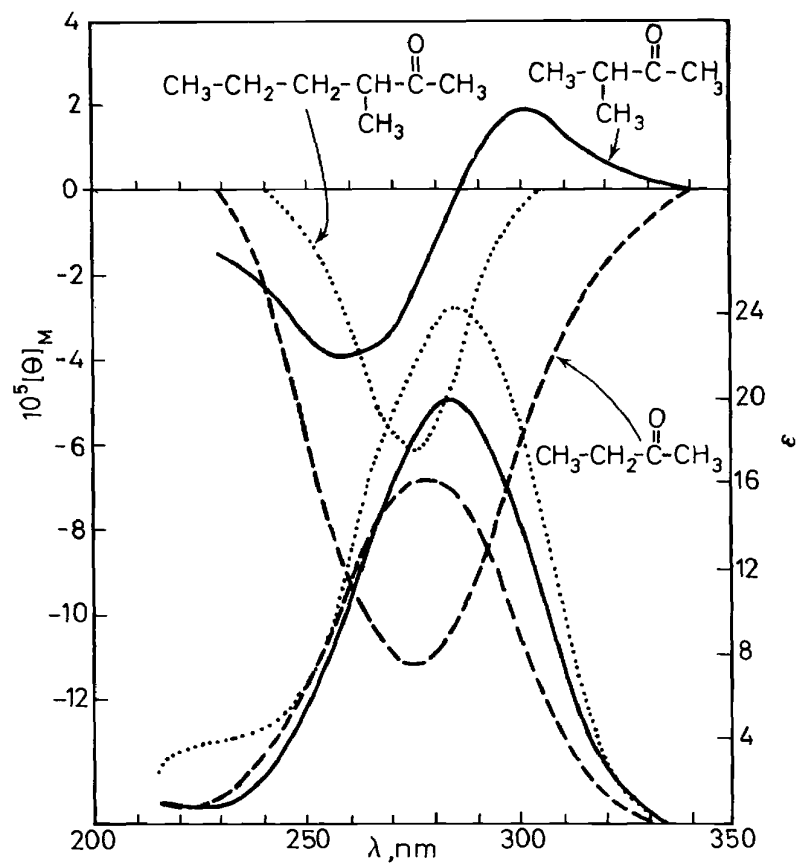

Figure 21. MCD and absorption spectra of 2-butanone (- - - ), 3-methyl-2-butanone (- - ), and 3-methyl-2-hexanone ( $\cdots)$ in cyclohexane. $[\theta]_{M}$ is normalized to 1 gauss.

this situation is that the effects exhibited by saturated carbonyl compounds are very small and require the use of particularly sensitive instruments in conjunction with superconducting magnets. For example, the values in Table 1 illustrate the range of magnitudes of the effects observed in the MCD spectra of some of the organic compounds cited in the present article.

Table 1. Range of MCD band magnitudes

\begin{tabular}{lccc}
\hline \multicolumn{1}{c}{ Compound } & Figure & $\lambda_{\max .}(\mathrm{nm})$ & {$[\theta]_{\mathrm{M}}$ (per unit field) } \\
\hline Mg(II) deuteroporphyrin IX dimethyl ester & 5 & 406 & +115 \\
{$[18]$ annulene } & 12 & 371 & +3.81 \\
purine & 14 & 267 & +0.26 \\
cyclohexanone & 20 & 312 & +0.000023 \\
\hline
\end{tabular}

Thus McCaffery et al. ${ }^{69}$ reported that MCD effects could be observed only for cyclobutanone (Figure 24), 2-bromocyclobutanone and acetophenone whereas the MCD effects exhibited by a number of cyclic and acyclic ketones were too small to be detected. As a result, when we were recently able to achieve a significant enhancement in the sensitivity of our 
Durrum-JASCO circular dichrometer, it was of considerable interest to reinvestigate the general question of the MCD of carbonyl compounds. Our interest in the MCD of carbonyl compounds has been a continuing one and is based on our earlier success ${ }^{1-3}$ with ORD and CD measurements of optically active compounds containing the carbonyl chromophore. Thus, we have been particularly interested in ascertaining the extent to which MCD may provide information about the structure of optically inactive (achiral) carbonyl compounds. Indeed, the first few compounds measured showed a variety of shapes and signs for the MCD bands associated with the singlet $\mathrm{n}-\pi^{*}$ transition in the $290 \mathrm{~nm}$ region.

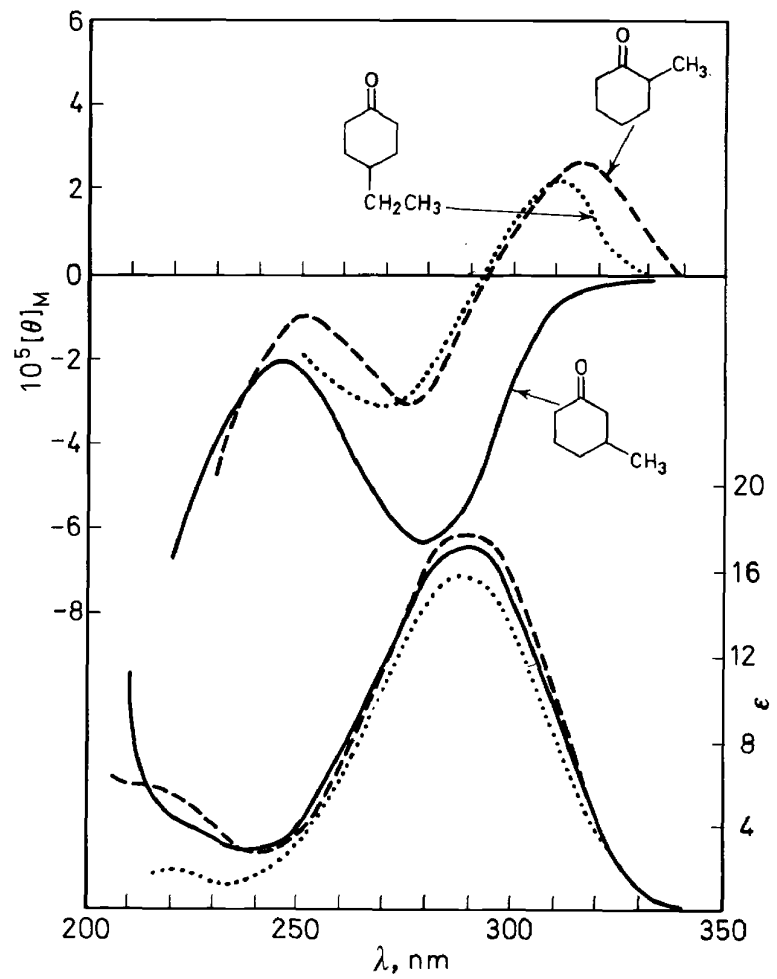

Figure 22. MCD and absorption spectra of 2-methylcyclohexanone (-- - ), 3-methylcyclohexanone (-), and 4-ethylcyclohexanone $(\cdots)$ in cyclohexane. $[\theta]_{M}$ is normalized to 1 gauss.

The MCD curves of four simple carbonyl compounds are shown in Figure 20 in order to illustrate the range of effects which have been found ${ }^{70,71}$. Acetone and adamantanone both show a single but oppositely signed MCD band at 275 and $295 \mathrm{~nm}$, respectively. For both compounds the maximum values in the MCD spectrum and in the absorption spectrum are in relatively close correspondence. Cyclohexanone shows three well-resolved positive components centred about $312 \mathrm{~nm}$ and a negative, but structureless, band at $275 \mathrm{~nm}$. The sign pattern is reversed in the case of 2,2-dimethylbutanal since the negative component is found at $302 \mathrm{~nm}$ and a much weaker positive 
component at $272 \mathrm{~nm}$. It is apparent from their curves that MCD is considerably more sensitive to the structural environment of the carbonyl group than had been supposed. As a consequence, it seemed imperative to assess empirically the level at which stereochemical information would appear ${ }^{71}$.

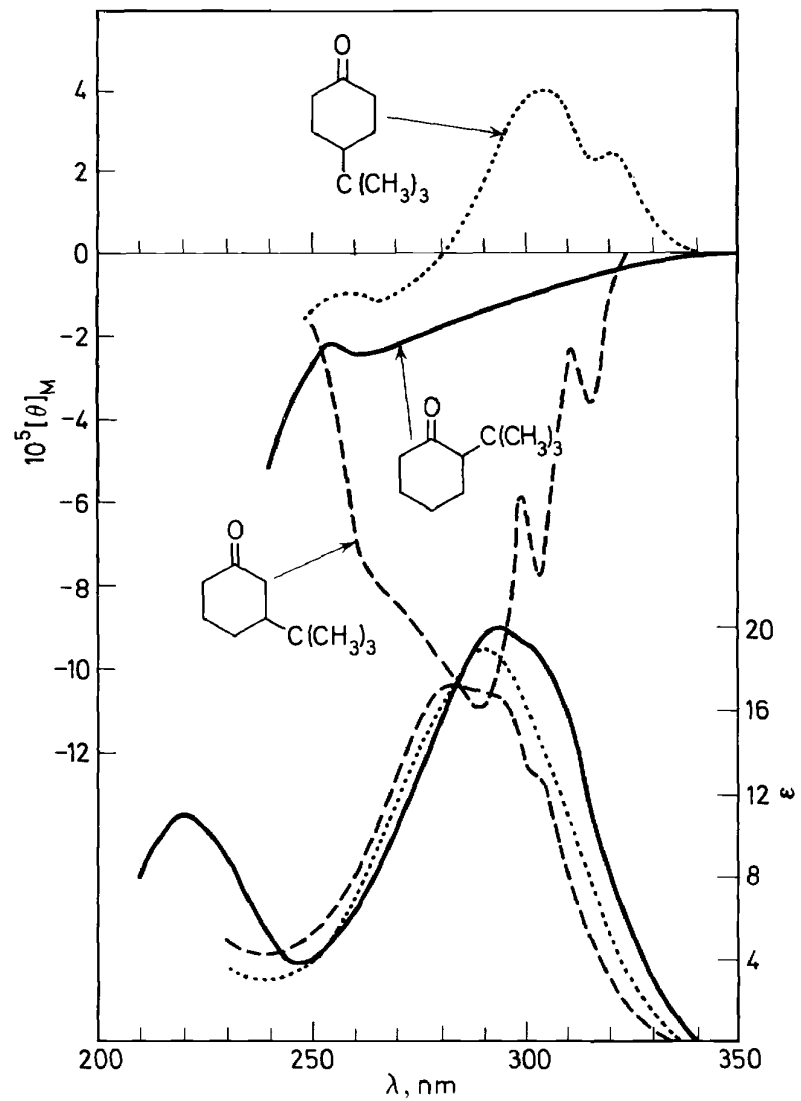

Figure 23. MCD and absorption spectra of 2-t-butyl-(-_-), 3-t-butyl-(----), and 4-t-butylcyclohexanone $(\cdots)$ in cyclohexane. $[\theta]_{M}$ is normalized to 1 gauss.

The MCD curves of several acyclic ketones are shown in Figure 21. The single negative MCD band exhibited by 2-butanone is characteristic for all of the $n$-alkanones measured irrespective of the number of carbon atoms as well as the position of the carbonyl group. Replacement of one or more $\alpha^{-}$or $\alpha^{\prime}$-hydrogen atoms may result in the appearance of two MCD bands as illustrated by the curve for 3-methyl-2-butanone. In some cases the positive component is not directly evident in the MCD spectrum. For example, the position of the MCD maximum with respect to the absorption maximum in the curve for 3-methyl-2-hexanone indicates the presence of a weakly positive band at longer wavelengths.

The MCD curves of several alkyl derivatives of cyclohexanone are shown in Figures 22 and 23 in order to illustrate the range of effects exhibited by 


\section{CARL DJERASSI, EDWARD BUNNENBERG AND DANNY ELDER}

alkyl substituents in the 2, 3 and 4 positions. The MCD curves (Figure 22) for 2-methyl- and 4-ethyl-cyclohexanone closely resemble that of cyclohexanone except for the lack of discernible fine structure in the positive band. In the spectra of 3-methyl- (Figure 22) and 3-t-butyl-cyclohexanone (Figure 23) only negative bands are evident. The weak and poorly defined curve (Figure 23) of 2-t-butylcyclohexanone suggests the presence of both positive and negative components which are of nearly the same energy and magnitude and hence effectively cancel each other. In the MCD spectrum of 4-t-butylcyclohexanone (Figure 23) two oppositely signed components are again evident; however, in this case the amplitude of the positive component is considerably greater than that of the negative one.

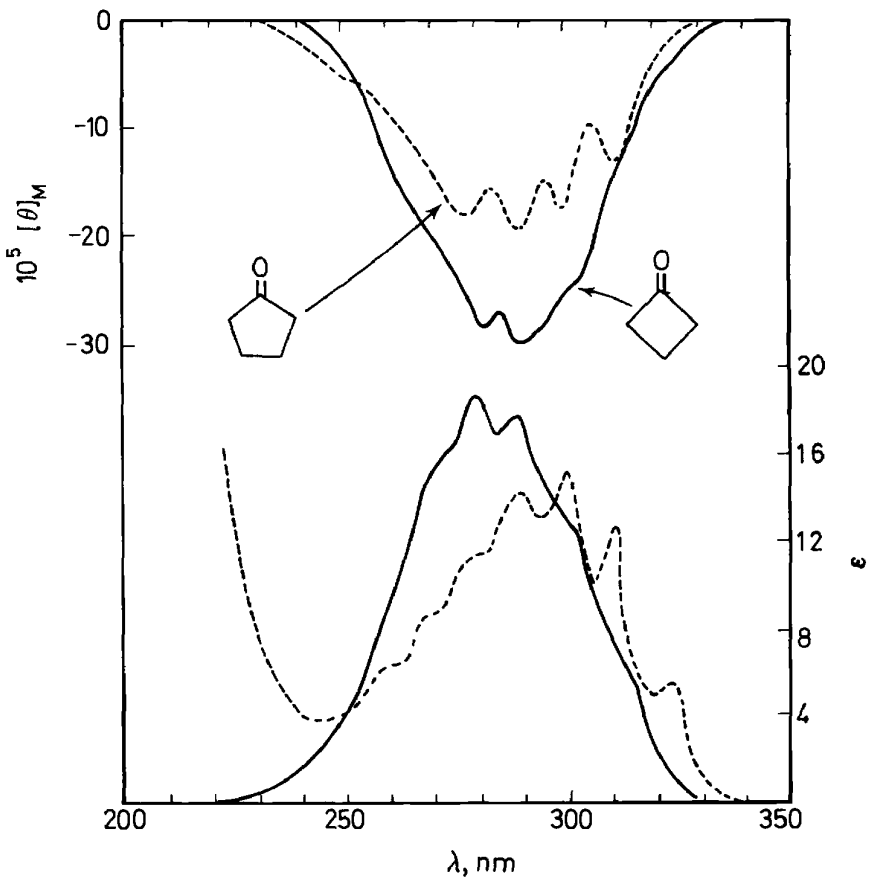

Figure 24. MCD and absorption spectra of cyclobutanone $(-)$ and cyclopentanone $(---)$ in cyclohexane. $[\theta]_{M}$ is normalized to 1 gauss.

In the unsubstituted cyclanone series shown in Figures 24 and 25 the multiplicity as well as the magnitude of the MCD bands depends on the ring size. In the case of cyclopentanone (Figure 24) each of the vibrational components in the absorption spectrum has its counterpart in the MCD spectrum. Cycloheptanone (Figure 25) shows two oppositely signed components as does cyclohexanone (Figure 20). Only single negative effects are evident in the curves (Figure 25) for cyclooctanone, cyclononanone, and cyclodecanone whereas cyclododecanone again shows two oppositely signed components. It is interesting that considerably more structure is found in the MCD curves of cyclooctanone and cyclodecanone than in their absorption curves. 
The results of a detailed analysis ${ }^{53}$ of the magnetic optical activity of the carbonyl chromophore indicate that the correlation between structure and MCD in the $n-\pi^{*}$ absorption band of aldehydes and ketones must be approached with caution. Perhaps the most general statement which can be made is that for the locally electric dipole forbidden $n-\pi^{*}$ transition of aldehydes and ketones ${ }^{73} \mathrm{MCD}$ is particularly sensitive to the ratio of the symmetry allowed part to that part which is locally forbidden but which is

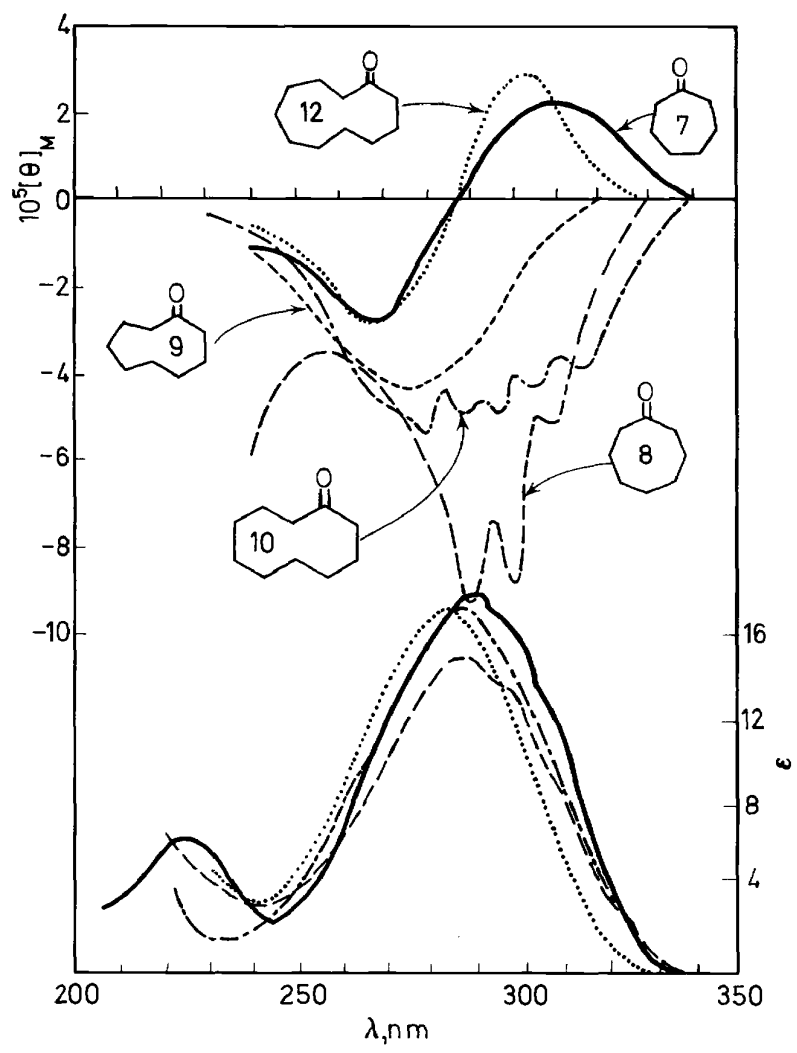

Figure 25. MCD and absorption spectra of cycloheptanone (- - ), cyclooctanone (-- - - ) cyclononanone $(---)$, cyclodecanone $(--\rightarrow)$, and cyclododecanone $(\cdots)$ in cyclohexane. $[\theta]_{M}$ is normalized to 1 gauss.

made allowed through non-totally symmetric vibrations. In the case of a ketone having $C_{2 v}$ symmetry (e.g. adamantanone), the $\mathrm{n}-\pi^{*}$ transition is allowed only by vibrational perturbations. By considering the factors which influence the mixing of the $n-\pi^{*}$ state with other nearby states (e.g. the $n-\sigma^{*}$ and $\pi-\pi^{*}$ states), relative selection rules can be established for the sign of the $B$ term associated with particular vibronic symmetries of the $n-\pi^{*}$ state. For symmetrical compounds, specific structures can, in principle, be correlated with the sign of the $B$ term by comparing which states are mixed through reduction in symmetry (to $C s, C s^{\prime}$ and $C_{2}$ ) with those which are mixed by vibrational perturbations. However, structural correlations may 
be obscured by the incursion of vibrational perturbations on an equal level with symmetry perturbations.

It is tempting to correlate the positive and negative components observed in the MCD spectrum of cyclohexanone (Figure 20) with the chair and twist conformations, respectively. However, a more careful analysis ${ }^{53}$ indicates that the positive structured components (Figure 20) are due to a vibrational perturbation of the same mode as is responsible for the positive effect observed for adamantanone (Figure 20). The negative component is attributed to contributions from the chair and twist conformations of cyclohexanone; however, vibrational perturbations must also be considered. For example, the single negative MCD band observed (Figure 20) for acetone would suggest that either its equilibrium conformation is lower than $C_{2 v}$ (i.e. $C_{2}$ ) or that, if $C_{2 v}$, the $\mathrm{n}-\pi^{*}$ transition is made allowed by a different vibrational perturbation than the one which is effective for adamantanone.

In summary, MCD is clearly a potentially important tool for spectroscopic assignments, for separation of certain overlapping absorption bands and for analytical purposes. In optically active compounds, CD and MCD may frequently offer complementary information and more routine measurements of MCD of optically active compounds are definitely indicated. The possible role of MCD for structural or conformational deductions among achiral substances is still uncertain, but our most recent observations with carbonyl compounds suggest that more extensive studies are certainly warranted.

\section{ACKNOWLEDGEMENT}

We are indebted to Mrs Ruth Records and Mr Gordon H. Scott for their technical assistance and to $\mathrm{Mr} \mathrm{A}$. M. Babcock for preparation of the figures. Indispensable financial assistance was provided by the National Institutes of Health, the National Science Foundation, NATO, and the Stanford Center for Materials Research.

\section{REFERENCES}

${ }^{1}$ C. Djerassi, Pure Appl. Chem. 2, 475 (1961).

2 C. Djerassi, Optical Rotatory Dispersion: Applications to Organic Chemistry, McGraw-Hill: New York (1960).

${ }^{3}$ C. Djerassi, Proc. Chem. Soc. 314 (1964).

4 P. Crabbé, Optical Rotatory Dispersion and Circular Dichroism in Organic Chemistry, Holden-Day; San Francisco (1965). The French version [Gauthier-Villars: Paris (1968)] covers the literature through early 1967.

${ }^{5}$ L. Velluz, M. Legrand and M. Grosjean, Optical Circular Dichroism, Verlag Chemie: Weinheim (1965).

6 G. Snatzke (ed.). Optical Rotatory Dispersion and Circular Dichroism in Organic Chemistry, Heyden and Son : London (1967).

${ }^{7}$ For nomenclature see C. Djerassi and W. Klyne, Proc. Chem. Soc. 55 (1957).

8 M. Faraday, Phil. Trans. Roy. Soc. London, 3 , 1 (1846).

9 M. Verdet, Ann. Chim. Phys. 41, $370(1854)$ and subsequent articles.

10 W. H. Perkin Sr, J. Chem. Soc. 41, 330 (1882) and subsequent articles.

11 T. M. Lowry, Optical Rotatory Power, chapters 12 and 35. Longman's Green : London (1935).

12 H. K. Wipf, J. T. Clerc and W. Simon, Helv. Chim. Acta, 51, 1051 and 1162 (1968). 


\section{ORGANIC CHEMICAL APPLICATIONS OF MAGNETIC CIRCULAR DICHROISM}

13 Leading references to analytical applications in this area may be found in a review by J. G. Dawber, Analyst, 89, 755 (1964).

14 R. W. Wood, Phil. Mag. [6], 10, 408 (1905).

15 A. Cotton and M. Scherer, C.R. Acad. Sci. Paris, 195, 1342 (1932).

16 A. Cotton and R. Servant, C.R. Acad. Sci. Paris, 214, 513 (1942).

17 V. E. Shashoua, J. Amer. Chem. Soc. 86,2109(1964). The data in a preliminary communication [idem ibid. 82, $5505(1960)]$ suffered from instrumental artifacts.

18 J. Winkler, Z. Analyt. Chem. 235, 18 (1968).

19 D. A. Schooley, E. Bunnenberg and C. Djerassi, Proc. Nat. Acad. Sci. Wash. 52, 579 (1965).

20 J. Badoz, chapter 22 in ref. 6.

21 D. A. Schooley, E. Bunnenberg and C. Djerassi, Proc. Nat. Acad. Sci. Wash. 56, 1377 (1966). A similar interest in superconducting magnets was also shown by other groups, e.g. P. N. Schatz, A. J. McCaffery, W. Suëtaka, G. N. Henning, A. B. Ritchie and P. J. Stephens. J. Chem. Phys. 45, 722 (1966).

22 See for instance A. Moscowitz, chapter 12 in ref. 2 and J. R. MacDonald and M. K. Brachman, Rev. Mod. Phys. 28, 393 (1956).

${ }^{23}$ See for instance W. Heller, J. Phys. Chem. 62, 1569 (1958).

24 A. D. Buckingham and P. J. Stephens, Ann. Rev. Phys. Chem. 17, 399 (1966).

25 P. N. Schatz and A. J. McCaffery, Quart. Rev. 23, 552 (1969).

26 R. Serber, Phys. Rev. 41, 489 (1932).

27 P. J. Stephens, W. Suëtaka and P. N. Schatz, J. Chem. Phys. 44, 4592 (1966).

28 R. E. Linder, $P h$. D.Thesis, Texas A and M University (1969).

29 V. E. Shashoua, J. Amer. Chem. Soc. 87, 4044 (1965).

30 V. E. Shashoua, Nature, London, 203, 972 (1964).

31 V. E. Shashoua, Arch. Biochem. Biophys. 111, 550 (1965).

32 M. V. Volkenstein, J. A. Sharonov and A. K. Shemelin, Nature, London, 209, 709 (1966).

${ }^{33}$ B. Briat, D. A. Schooley, R. Records, E. Bunnenberg and C. Djerassi, J. Amer. Chem. Soc. 89, 6170 (1967).

${ }^{34}$ C. Houssier and K. Sauer, J. Amer. Chem. Soc. 92,779 (1970).

${ }^{35}$ G. W. Hodgson, E. Bunnenberg, B. Halpern, E. Peterson, K. A. Kvenvolden and C. Ponnamperuma, in Proceedings of the Appollo 11 Lunar Science Conference (edited by A. A. Levinson), p 1829. Pergamon Press: New York (1970).

36 B. Briat and C. Djerassi, Bull. Soc. Chim. France, 135 (1969).

37 E. A. Dratz, Ph.D. Thesis, University of California: Berkeley (1966).

See also L. J. Boucher, J. Amer. Chem. Soc. 92, 2725 (1970).

38 P. S. Pershan, M. Gouterman and R. L. Fulton, Molec. Phys. 10, 397 (1966).

39 J. E. Falk, Porphyrins and Metalloporphyrins. Elsevier: New York (1964).

40 W. T. Simpson, J. Chem. Phys. 17, 1218 (1949).

${ }^{41}$ C. Weiss, H. Kobayashi and M. Gouterman. J. Molec. Spectrosc. 16, 415 (1965).

42 V. E. Shashoua in Hemes and Hemoproteins (B. Chance, R. W. Estabrook, and T. Yonetani, ed.) p 93. Academic Press : New York (1966).

43 P. J. Stephens, P. N. Schatz, A. B. Ritchie and A. J. McCaffery. J. Chem. Phys. 48, 132 (1968).

44 P. J. Stephens, Chem. Phys. Letters, 2, 241 (1968).

45 B. Briat, D. A. Schooley, R. Records, E. Bunnenberg and C. Djerassi, J. Amer. Chem. Soc. 89, 7062 (1967).

46 M. Gouterman and G. Wagniere, J. Chem. Phys. 36, 1188 (1962).

47 M. Gouterman and G. Wagniere, Tetrahedron Letters, 22 (1960).

${ }^{48}$ F. L. Hirshfelder and D. Rabinovich, Acta Cryst. Camb. 19, 227, 235 (1965).

49 L. Salem, The Molecular Orbital Theory of Conjugated Systems, W. A. Benjamin : New York (1966).

50 B. Briat, D. A. Schooley, R. Records, E. Bunnenberg, C. Djerassi and E. Vogel, J. Amer. Chem. Soc. 90, 4691 (1968).

51 J. G. Foss and M. E. McCarville, J. Chem. Phys. 44, 4350 (1966).

52 J. G. Foss and M. E. McCarville, J. Amer. Chem. Soc. 89, 30 (1967).

${ }^{53}$ L. Seamans and A. Moscowitz, Private communication.

54 B. Briat and M. Le Liboux, C.R. Acad. Sci. Paris, 262 (C), 803 (1066).

55 I. Tinoco Jr and C. A. Bush, Biopolymers Symposia No. 1, 235 (1964).

56 W. Voelter, R. Records, E. Bunnenberg and C. Djerassi, J. Amer. Chem. Soc. 90, 6163 (1968).

57 W. Voelter, G. Barth, R. Records, E. Bunnenberg and C. Djerassi, J. Amer. Chem. Soc. 91, 6165 (1969). 
58 D. L. Elder, E. Bunnenberg, C. Djerassi, M. Ikehara and W. Voelter, Tetrahedron Letters, 727 (1970).

59 M. E. McCarville, Ph.D. Thesis, Iowa State University (1967).

${ }^{60}$ D. W. Miles, R. K. Robins and H. Eyring, Proc. Nat. Acad. Sci. Wash. 57, 1138 (1967).

61 D. W. Miles, R. K. Robins and H. Eyring, J. Phys. Chem. 71, 3931 (1967).

62 G. Tollin, Biochemistry, 7, 1720 (1968).

63 J. L. Fox, S. P. LaBerge, K. Nishimoto and L. S. Forster, Biochim. Biophys. Acta, 136, 544 (1967).

${ }^{64}$ D. I. Marlborough, D. O. Hall and R. Cammack, Biochem. Biophys. Res. Commun. 35, 410 (1969).

${ }^{65}$ E. Bayer, W. Voelter, P. Krauss, E. Bunnenberg and C. Djerassi, In preparation.

66 B. Briat, M. Billardon and J. Badoz, C.R. Acad. Sci. Paris, 256, 3440 (1963).

${ }^{67}$ B. Briat, C.R. Acad. Sci., Paris, 258, 2788 (1964).

68 J. Winkler, Chem. Commun. 648 (1968).

69 A. J. McCaffery, G. N. Henning, P. N. Schatz, A. B. Ritchie, H. P. Perzanowski, O. R. Rodig, A. W. Norvelle Jr and P. J. Stephens, Chem. Commun. 520 (1966).

70 G. Barth, E. Bunnenberg and C. Djerassi, Chem. Commun. 1246 (1969).

${ }^{71}$ G. Barth, E. Bunnenberg, C. Djerassi, D. L. Elder and R. Records. Symposium of the Faraday Society, No. 3, 49 (1969).

72 P. J. Stephens, Ph.D. Thesis, Oxford University (1964).

73 J. A. Pople and J. W. Sidman, J. Chem. Phys. 27, 1270 (1957).

\section{Note added in proof}

The following additional papers on magnetic circular dichroism are of some relevance to our review:

74 B. P. Atanasov, M. V. Volkenstein and Y. A. Sharonov, Molec. Biol. (USSR), 3, 696 (1969). 'Dispersion of magnetooptical rotation of ferri-myoglobin and hemin and their complexes with some ligands'.

75 B. P. Atanasov, M. V. Volkenstein and Y. A. Sharanov, Molec. Biol. (USSR), 3, 804 (1969). 'Investigation of the magnetooptical rotatory dispersion of the hemin and heme complexes with low-molecular ligands'.

76 B. P. Atanasov, M. V. Volkenstein and Y. A. Sharonov, Molec. Biol. (USSR), 3, 518 (1969). 'Investigations of the modified cytochrome $\mathrm{c}$ with myoglobin-like properties by the magnetooptical rotation 'method'.

77 B. Briat, Méthodes Physiques d'Analyse (GAMS), 6, 19 (1970). A review.

78 P. J. Stephens, J. Chem. Phys., 52, 3489 (1970). 'Theory of magnetic circular dichroism'.

79 H. Kobayashi, M. Shinizu and I. Fujita, Bull. Chem. Soc. Japan, 43, 2335 (1970). 'Magnetic circular dichroism of iron tetraphenylporphins'.

80 M. H. Perrin, M. Gouterman and C. L. Perrin, J. Chem. Phys. 50, 4137 (1969). A discussion of the MCD spectra of metalloporphyrins particularly with regard to the 'anomalous' Zeeman spectra reported by M. Malley, G. Feher and D. Manzerall, J. Molec. Spectrosc. 26, 320 (1968).

81 J. C. Sutherland, D. Axelrod and M. P. Klein, J. Chem. Phys. (submitted). 'Zeeman effect in porphyrims: zero-field splitting of the excited electronic states'.

82 G. Barth, R. Records, E. Bunnenberg, C. Djerassi and W. Voelter, J. Amer. Chem. Soc. (submitted). 'The determination of tryptophan in proteins'.

${ }^{83}$ U. Weber, E. Bunnenberg, R. Cammack, C. Djerassi, R. Flohé, G. Thomas and W. Voelter, Biochem. (submitted). 'A study of purified bovine erythrocuprein'.

${ }^{84}$ A. Abu-Shumays and J. J. Duffield, Applied Spectrosc. 24, 67 (1970). "Measurement of magnetic circular dichroism as a modified spectropolarimeter'. 